\title{
Evaluating Social Media as a Medium of Private Communication Through Steganographic Images
}

\author{
Kendall Weldon Coles \\ West Virginia University, kwcoles@mix.wvu.edu
}

Follow this and additional works at: https://researchrepository.wvu.edu/etd

Part of the Digital Communications and Networking Commons, and the Social Media Commons

\section{Recommended Citation}

Coles, Kendall Weldon, "Evaluating Social Media as a Medium of Private Communication Through Steganographic Images" (2021). Graduate Theses, Dissertations, and Problem Reports. 8020. https://researchrepository.wvu.edu/etd/8020

This Thesis is protected by copyright and/or related rights. It has been brought to you by the The Research Repository @ WVU with permission from the rights-holder(s). You are free to use this Thesis in any way that is permitted by the copyright and related rights legislation that applies to your use. For other uses you must obtain permission from the rights-holder(s) directly, unless additional rights are indicated by a Creative Commons license in the record and/ or on the work itself. This Thesis has been accepted for inclusion in WVU Graduate Theses, Dissertations, and Problem Reports collection by an authorized administrator of The Research Repository @ WVU. For more information, please contact researchrepository@mail.wvu.edu. 


\title{
EVALUATING SOCIAL MEDIA AS A MEDIUM OF PRIVATE COMMUNICATION THROUGH STEGANOGRAPHIC IMAGES
}

\author{
Kendall Weldon Coles \\ Thesis submitted to the Statler College of Engineering and Mineral Resources \\ at West Virginia University \\ in partial fulfillment of the requirements for the degree of \\ Master of Science in \\ Computer Science \\ Roy Nutter, Ph.D., Chair \\ Katerina Goseva-Popstojanova, Ph.D. \\ Frances Van Scoy, Ph.D. \\ Lane Department of Computer Science and Electrical Engineering
}

Morgantown, West Virginia

2021

Keywords: Steganography, Communication, Social Media, Hidden, Secret, Image Copyright 2021 Kendall Coles 


\title{
Abstract \\ EVALUATING SOCIAL MEDIA AS A MEDIUM OF PRIVATE COMMUNICATION THROUGH STEGANOGRAPHIC IMAGES
}

\begin{abstract}
Kendall Coles
Social media is a vastly used communication tool with billions of users worldwide. These social networks provide users the ability to share their ideas and thoughts through the messages, videos, and images that they post. The images that are shared can possibly be embedded with private messages through the use of a steganographic tool. The messages are embedded in a fashion that doesn't change the visual appearance of an image. This allows for these types of images to hide in plain sight, which creates the possibility of someone communicating privately in a public social media setting. This project proved how that it is possible, with an experiment that tested the image stego tools DIIT, Steghide, OutGuess, and ExifTool on Reddit and Twitter, using a method developed for sharing steganographic images to social media websites.
\end{abstract}




\section{Acknowledgements}

I would first like to thank Dr. Nutter for giving me the opportunity to continue my education as a graduate research assistant and for his guidance and support. I would also like to thank my committee members, Dr. Van Scoy and Dr. Goseva-Popstojanova, for their patience and support while I was conducting research.

Lastly, I would like to thank my family and friends. I wouldn't be in the position that I'm in today without them. 


\section{Table of Contents}

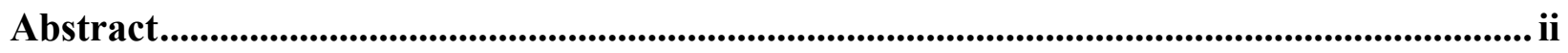

Acknowledgements .......................................................................................................................... iii

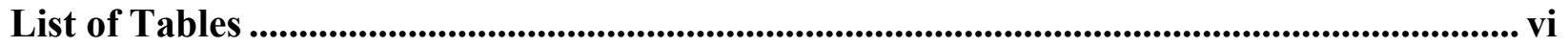

List of Figures..................................................................................................................... vii

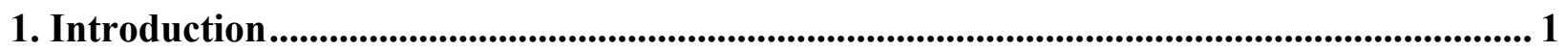

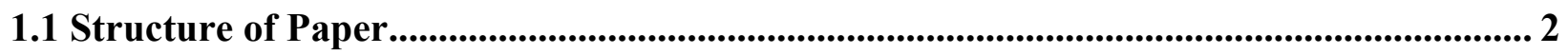

2. Background Information.................................................................................................. 2

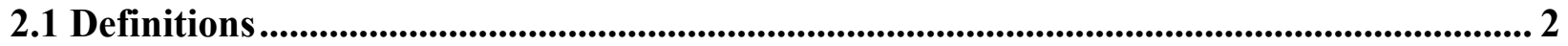

2.2 Digital Images...................................................................................................................... 3

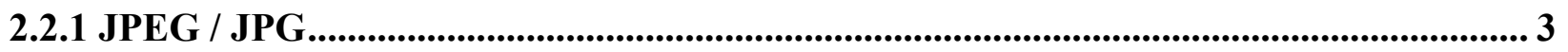

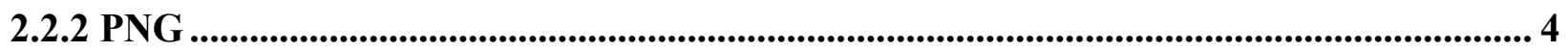

2.3 Cryptography versus Steganography _........................................................................ 4

2.5 Image Steganography Techniques.............................................................................................. 5

2.5.1 Spatial Domain ........................................................................................................................ 5

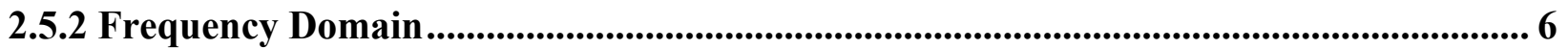

2.5.3 Structure Based ............................................................................................................................ 6

2.5.4 Steganography Example.................................................................................................... 6

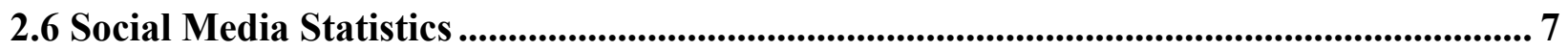

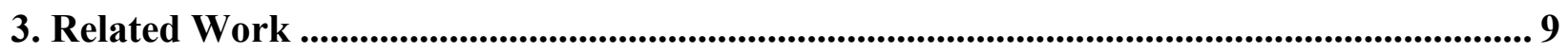

3.1 Evaluation of Impact of Image processing on Stego Images................................................. 9

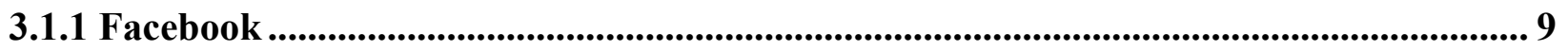

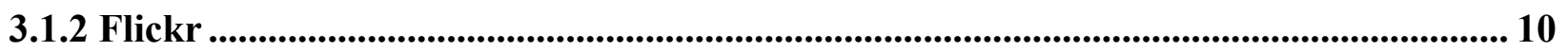

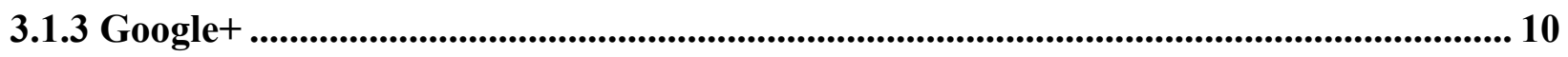

3.1.4 Twitter.................................................................................................................................... 10

3.2 StegHash: New Method for Information Hiding in Open Social Networks ....................... 10

3.3 Case Study on Steganography in Social Media ......................................................................... 11

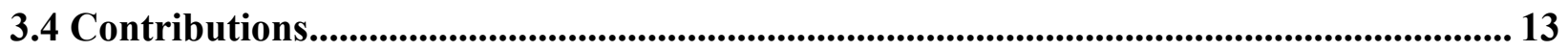

4. Problem Statement................................................................................................................... 13

5. Description of Pilot Experiment ...................................................................................................... 14

6. Description of Experiment ................................................................................................................ 14

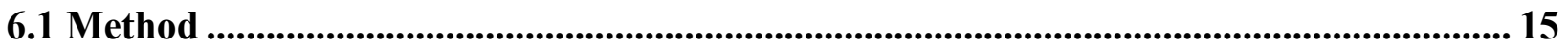




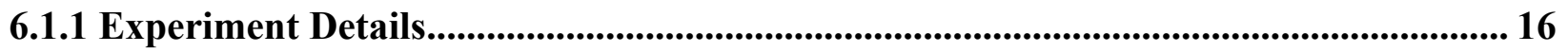

6.1.2 Digital Invisible Ink Toolkit (DIIT) .................................................................................. 17

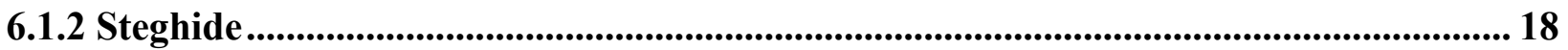

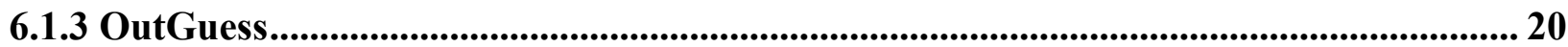

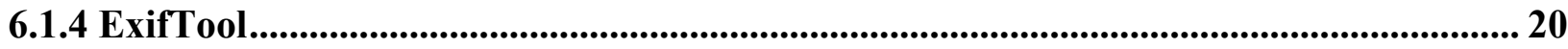

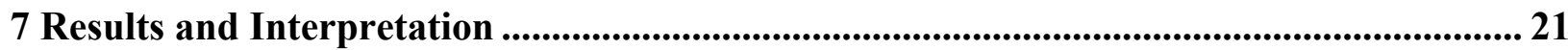

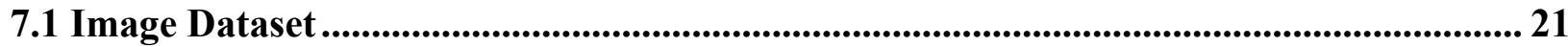

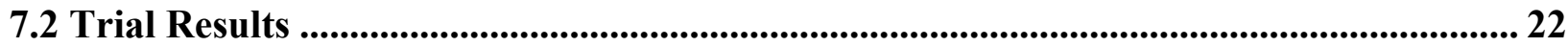

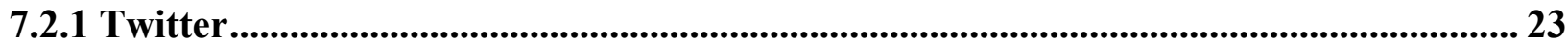

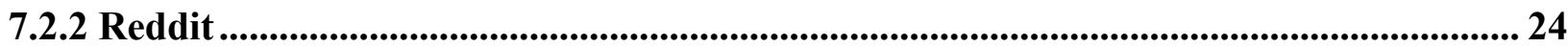

7.2.3 Key Takeaways and Updated Table................................................................................ 25

7.3 Compression Ratios ............................................................................................................. 25

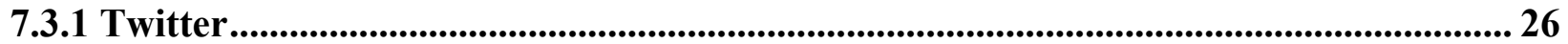

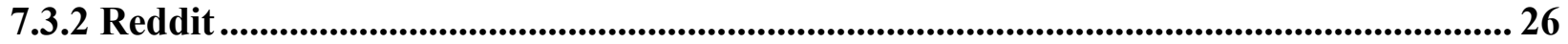

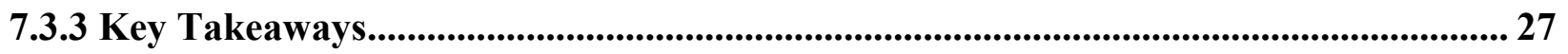

7.4 MSE of Uploaded and Downloaded Images ........................................................... 27

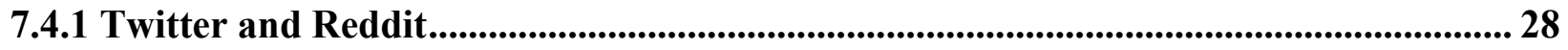

7.4.3 Key Takeaways....................................................................................................................... 31

7.5 Quantization Tables............................................................................................................................... 31

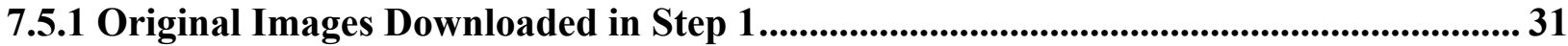

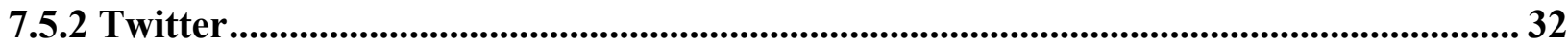

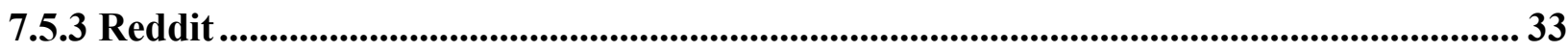

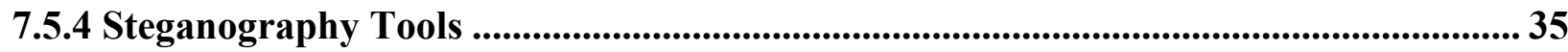

7.5.5 Key Takeaways..................................................................................................................................... 35

8.Threats to Validity ............................................................................................................. 36

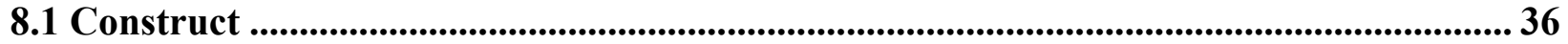

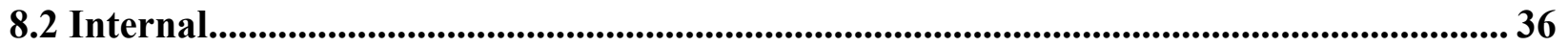

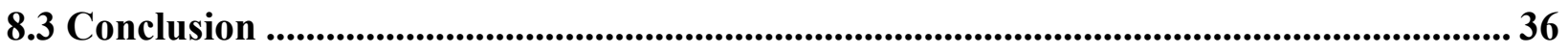

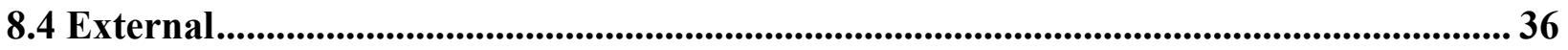

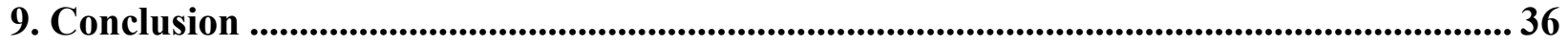

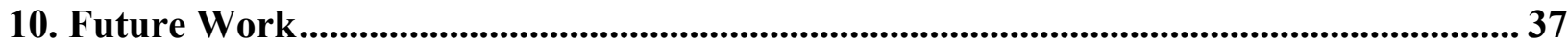

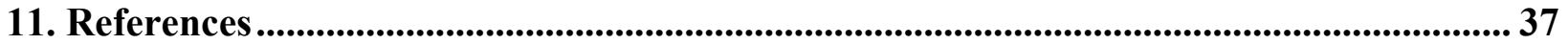




\section{List of Tables}

TABLE 1: IMAGE METADATA

TABLE 2: INTERNET USAGE IN A MINUTE [18]

TABLE 3: SOCIAL NETWORKS RANKED 1-17 [4]

TABLE 4: KEY DEFINITIONS FOR TABLE 5 [1]

TABLE 5: SUMMARY OF CASE STUDY AND EXPERIMENT [1]

TABLE 6: STEGO TOOL LINKS

TABLE 7: ALL COMBINATIONS FOR THE EXPERIMENT

TABLE 8: RESULTS FROM THE EXPERIMENTS USING EVERY COMBINATION

TABLE 9: RESULTS FOR ALL TWITTER IMAGES

TABLE 10: TWITTER RESULTS BELOW THRESHOLD

TABLE 11: TWITTER RESULTS ABOVE THRESHOLD

TABLE 12: REDDIT RESULTS FOR ALL IMAGES

TABLE 13: UPDATED TABLE WITH NEW RESULTS

TABLE 14: QUALITY FACTOR FREQUENCY OF ORIGINAL IMAGES

TABLE 15: QUALITY FACTOR FOR TWITTER IMAGES

TABLE 16: QUALITY FACTOR FREQUENCY FOR REDDIT IMAGES 


\section{List of Figures}

FIGURE 1: EXAMPLE OF LOSSY COMPRESSION [13] 3

FIGURE 2: ROT13 CIPHER EXAMPLE [8]

FIGURE 3: MULTIPLE LAYER CRYPTOGRAPHY EXAMPLE [8][16]

FIGURE 4: LSB METHOD EXAMPLE [26][28] 26

FIGURE 5: STEGHASH METHOD [24] 11

FIGURE 6: DEVELOPED METHOD USED IN EXPERIMENT

FIGURE 7:MODIFIED METHOD FOR POSTING STEGANOGRAPHIC IMAGES TO SOCIAL MEDIA [1]

FIGURE 8: ENCODE WINDOW $\quad 18$

FIGURE 9: DECODE WINDOW $\quad 18$

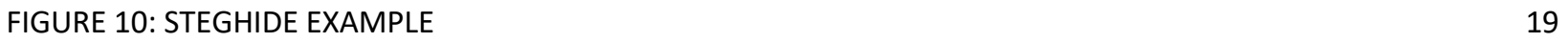

FIGURE 11: OUTGUESS EXAMPLE $\quad 20$

FIGURE 12: EXIFTOOL EXAMPLE $\quad 21$

FIGURE 13: IMAGE DATASET PRODUCED FROM STEP 1 OF THE METHOD 22

FIGURE 14: COMPRESSION RATIOS OF TWITTER IMAGES 26

FIGURE 15: COMPRESSION RATIOS OF REDDIT IMAGES 27

FIGURE 16: MSE EQUATION 28

FIGURE 17: MSE OF TWITTER AND REDDIT IMAGES 28

FIGURE 18: MSE OF TWITTER AND REDDIT DIIT IMAGES 229

FIGURE 19: MSE OF TWITTER AND REDDIT STEGHIDE IMAGES 29

FIGURE 20: MSE OF TWITTER AND REDDIT OUTGUESS IMAGES

FIGURE 21: MSE OF TWITTER AND REDDIT EXIFTOOL IMAGES

FIGURE 22: TABLE O AND 1 FOR OUTGUESS IMAGES [21] 


\section{Introduction}

Steganography is the practice of hiding data within other data. The hidden information itself is usually concealed in a fashion that is undetectable. The purpose of steganography is to provide secret communication between two or more parties or hide the transmission of private data. The different types of steganography include network, image, audio, video, and linguistic. Therefore, the embedded message itself or carrier of the embedded message can be a text, image, video, or audio file. Image steganography uses a cover image as the carrier of the secret data. There are many free tools available for download online (some examples are included in table 6) that can be used to create this type of steganography (stego images). Once these images are produced, they can be shared online to online to popular social media websites like Facebook and Twitter, allowing for mass distribution of a message. Since plenty of photos are posted on social media each day, stego images can blend in with normal images. This makes social media an attractive medium for this method of private communication [1][2].

In this paper, an established method [1] for posting image steganography on social media websites will be further tested for its effectiveness. [17] 


\subsection{Structure of Paper}

In section 2 of the paper, background information related to steganography is discussed. Section 3 covers the related works that have been completed in the past relating to social media and steganography and it also discusses my planned contributions. Section 4 includes the problem statement, which explains the purpose of the research. Section 5 details the pilot experiment and its results. Section 6 details the experiment conducted after the pilot experiment and its results are discussed in section 7. Section 8 describes the threats to validity of making a correct conclusion. The conclusion and future works are in sections 9 and 10. Lasty, the references used are included in section 11 .

\section{Background Information}

\subsection{Definitions}

Steganography - The practice of hiding data within data. Data can be an image, message or file[5]

Stego - Short for steganography

Stego Tool - Tool used to create steganographic images

Stego Image - Image that contains embedded data

Cover Image - Image that is used to create steganographic image

Pixel (Picture Element) - A digital image is comprised of pixels. In an RGB color space, there are three bytes per pixel

Bit - 0 or 1

Byte -8 bits

JPEG (Joint Photographic Experts Group) - A computer file format for the lossy compression and storage of digital images [12]

PNG (Portable Network Graphics) - A computer file format for the lossless compression of digital images

LSB (Least Significant Bit) - The bit farthest to the right, holding the least amount of value in a binary number 
Metadata or EXIF Data (Exchangeable Image File Format) - Descriptive information that is embedded in an image. Examples include make and model of the camera that took the photo DIIT - Digital Invisible Ink Toolkit

Chrominance - Color of pixel

Luminance - Brightness of a pixel

\subsection{Digital Images}

\subsubsection{JPEG / JPG}

JPEG is a commonly used image file format that utilizes lossy compression.

Lossy compression is a data encoding method that allows for some details to be approximated and some partial data to be discarded. This makes it possible to reduce the size of an image without a noticeable change in its appearance, which is beneficial for storage and transmission of images. The tradeoff is showcased in figure 1, with the quality of the image being directly related to the amount of reduction or information loss. JPEGs have been shown to be able to use 10:1 to 20:1 compression ratio without any major visual difference [14]. The best compression ratio overall is 10:1 since it is able to produce images that preserve visual image quality [14].

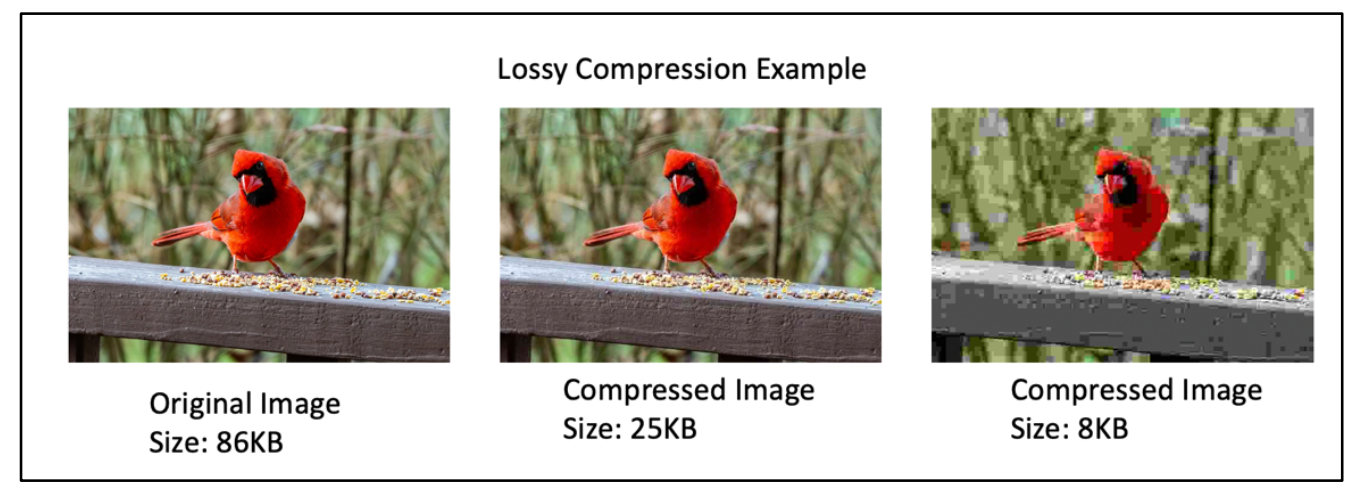

Figure 1: Example of Lossy Compression [13]

The JPEG compression process takes 6 steps if the image is in a RGB color space ( 3 bytes per pixel). The first stage converts the image from a RGB color space to $\mathrm{YCbCr}$ space, which separates the luminance and chrominance or each pixel. The image is then down sampled and split into $8 \times 8$ blocks of pixels. In the next stage a Discrete Cosine Transform is applied to each block. The quantization stage then occurs (where lossy compression takes place). Lastly entropy coding is used to produce a JPEG image [20]. 
In the quantization step, the device or software that is producing the image will use a set of commonly used quantization tables(most images have 1-4 tables). These tables contain 64 values $(8 \times 8)$ that range from $0-65,535$. The lower the value the less amount of data loss. The quantization tables (published by Independent JPEG Group) contain a table of values that are regularly used. These are also are scaled by a certain quality factor (ranging 0-100), that affects the quality of an image. These tables can be used to identify the type of camera or software that was used to create a particular image [20].

\subsubsection{PNG}

PNG is an image format that uses lossless data compression. When an image is reassembled using lossless compression, the quality does not change. This method of compression can be used for important data files that cannot be approximated (high quality audio files for example). PNGs also can usually have compression ratios from 1.5:1 to 3:1 [23].

\subsection{Cryptography versus Steganography}

Cryptography is the practice of encoding and decoding messages through the use of codes and keys. [15] When it is used a cipher text is produced using a particular algorithm. The cipher text can only be decoded back to the original text if the receiver of the message knows the algorithm that was used. Cryptography is used for secure network communications, disk encryption, authentication, and data integrity checking.

A simple example of a cryptography algorithm is the ROT13 cipher (pictured in figure 2). Let's say two parties were trying to exchange a secret message or passphrase using this cipher. The transmitter wants to send the message "Let's Go Mountaineers". The cipher text would then be "Yrg'f tb Zbhagvnarref", which is unintelligible [8]. (in a realistic scenario this would be a relatively easy cipher to decrypt). Before the message is sent, the transmitter informs the receiver of what algorithm is being used. The receiver of the message can use this knowledge to retrieve the secret message [17]. In ROT13, which is a substitution cipher, the alphabet being used is shifted a certain number of places. In this case the alphabet is shifted 13 places, meaning every occurrence of " $\mathrm{A}$ " is replaced by " $\mathrm{N}$ ", and every occurrence of " $\mathrm{B}$ " is replaced by "O", and so on [8]. 
Even if the communication was intercepted in the process of being sent and received, the outside party would still have to figure out what cryptography algorithm is being used, which can be a difficult process. Some algorithms like AES (Advanced Encryption Standard), RSA (RivestShamir-Adleman), and Twofish are almost impossible to decrypt even if the outside party knows they are being used.

Plain Text Cipher Text

"ABCDEFJHIJKLMNOPQRSTUVWXYZ" $\rightarrow$ ROT13 "NOPQRSWUVWXYZABCDEFGHIJKLM"

Figure 2: ROT13 Cipher Example [8]

One major difference between cryptography and steganography is that steganography hides the fact that secret communication is taking place. In steganography the cover file that has the embedded message appears to be normal. This allows secret messages to be transmitted in plain sight. Cryptography, on the other hand, only encrypts the secret message, as it does not hide any act of communication. Steganography and Cryptography can both add another layer of protection to their private message by using multiple layers of different algorithms. For example, in steganography the embedded secret message can also be encrypted with a cipher. In cryptography, the cipher text can be encrypted again using another cryptography algorithm.

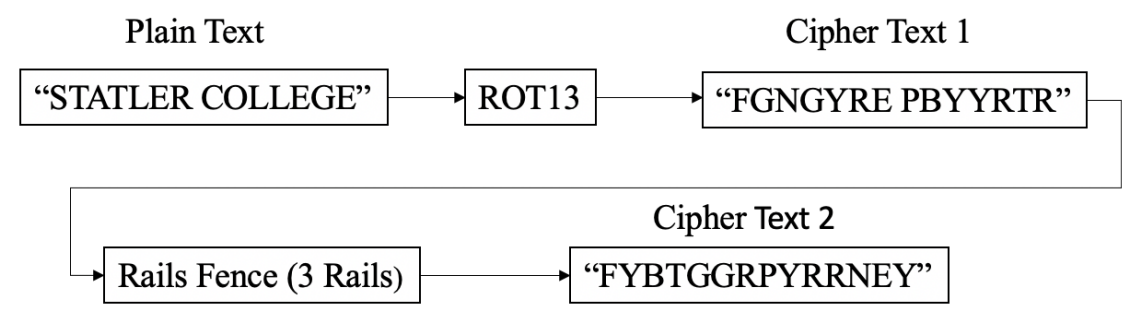

Figure 3: Multiple Layer Cryptography Example [8] [16]

\subsection{Image Steganography Techniques}

\subsubsection{Spatial Domain}


This technique usually modifies the LSB of multiple pixels to hide secret messages [10]. These minor changes to pixels are not easily recognized by the human eye. This is one of the least complex methods used [2][17].

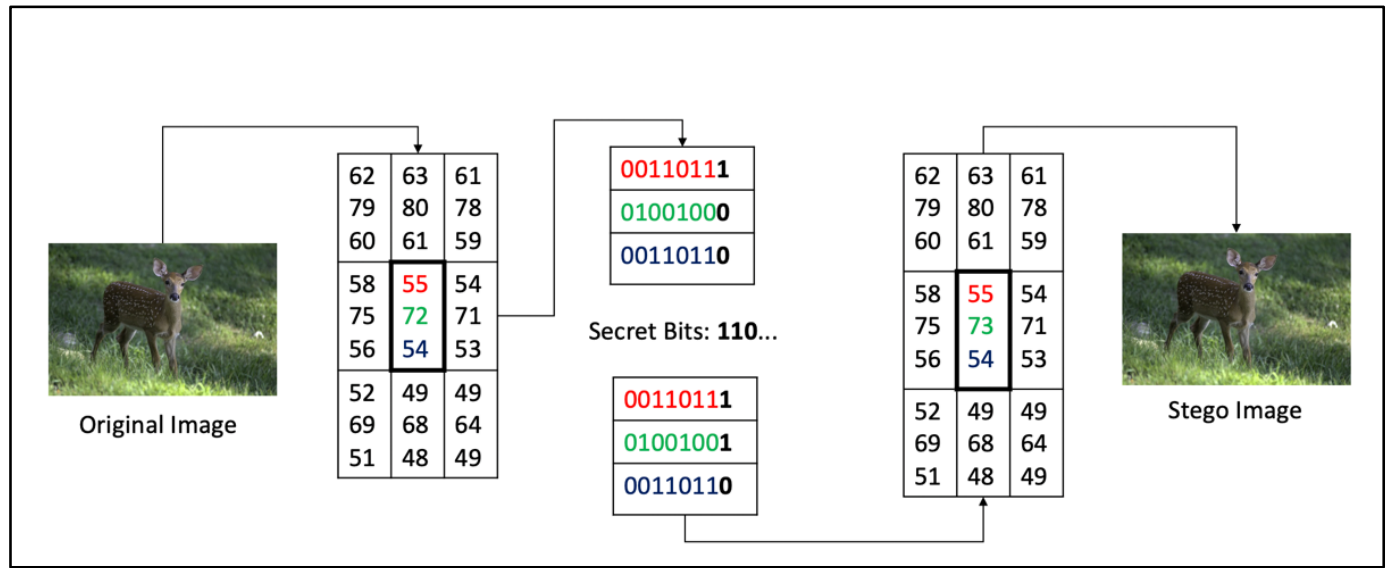

Figure 4: LSB Method Example [26][28]

\subsubsection{Frequency Domain}

This technique modifies the LSBs of discrete cosine transform coefficients [2]. Discrete cosine transform represents an image by a sum of varying cosine functions [11][17].

\subsubsection{Structure Based}

This method usually hides data by modifying an image's EXIF data [2][17].

\subsubsection{Steganography Example}

As discussed before, stego images have the ability to hide in plain sight. Figures 5, and 6 show how that idea is possible. Although figure 5 and 6 appear to be the same image visually, figure 5 is a normal image, and figure 6 is a stego image with the message "Let's go Mountaineers!" embedded as a text file. The stego image was created using the Digital Invisible Ink Toolkit with the BattleSteg algorithm. It's a free tool available for download online [9], and it used in the experiment and discussed in more detail in section 6.1 . 


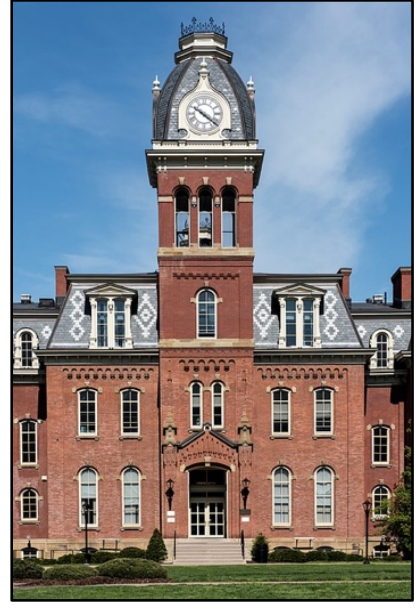

Figure 5: Cover Image [22]

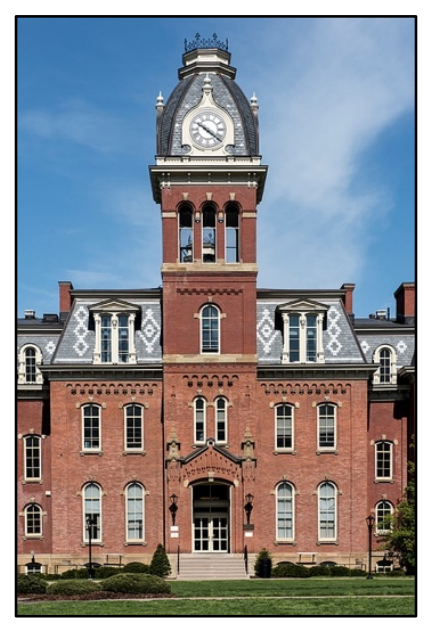

Figure 6: Stego Image [22]

Table 1: Image Metadata

\begin{tabular}{|l|l|l|l|}
\hline Figure & Dimensions & File Size & MD5 Hash \\
\hline 5 & $427 \times 640$ pixels & $115 \mathrm{~KB}$ & 2cd08a7b1f57182258a39cac54eec955 \\
\hline 6 & $427 \times 640$ pixels & $569 \mathrm{~KB}$ & 23a58f4b5a873dc9ba8a48dbc1996cc1 \\
\hline
\end{tabular}

As seen in the table there is a major discrepancy between the image's sizes and their generated MD5 hashes. Generating hashes is a great way to check the integrity of a file. If there is a change in the file, hash of the file will change [17].

Another example of steganography involves hiding invisible text onto images. The text can only be made visible by changing filters and color schemes with a tool such as GIMP or StegSolve. This tactic is usually used to add hidden watermarks to images.

\subsection{Social Media Statistics}

One of the reasons that social media appears to be an attractive medium to share steganographic images is its volume of active users. The amount of activity from these users makes it more difficult to discover that steganography is being used. A study conducted in 2020 revealed 
amount of social media activity that occurs in in just one minute (summarized in table 2). Most of the activity revolves around instant messaging and streaming content, but many image uploads and user engagement occur as well. [18]

Table 2: Internet Usage in a Minute [18]

\begin{tabular}{|l|l|}
\hline Activity & Amount per minute \\
\hline Messages shared by WhatsApp Users & $41,666,667$ \\
\hline People Engaging with Reddit Content & 479,452 \\
\hline Stories posted by Instagram Users & 347,222 \\
\hline Messages Shared by Facebook Users & 150,000 \\
\hline Photos uploaded by Facebook Users & 147,000 \\
\hline $\begin{array}{l}\text { Clicks on business profile ads on } \\
\text { Instagram }\end{array}$ & 138,889 \\
\hline Tik Tok Installs & 2,704 \\
\hline $\begin{array}{l}\text { Hours of video uploaded by YouTube } \\
\text { users }\end{array}$ & 500 \\
\hline
\end{tabular}

In 2021, there was another study conducted to find the most popular social media networks in the world. It revealed that Facebook is still the most popular social network with YouTube in second [4].

Table 3: Social Networks Ranked 1-17 [4]

\begin{tabular}{|l|l|l|}
\hline Rank & Social Network & $\begin{array}{l}\text { Active Users } \\
\text { in Millions }\end{array}$ \\
\hline 1 & Facebook & 2740 \\
\hline 2 & YouTube & 2291 \\
\hline 3 & WhatsApp & 2000 \\
\hline 4 & Facebook Messenger & 1300 \\
\hline 5 & Instagram & 1221 \\
\hline 6 & Weixin / WeChat & 1213 \\
\hline 7 & Tik Tok & 689 \\
\hline 8 & QQ & 648 \\
\hline 9 & Douyin & 600 \\
\hline
\end{tabular}




\begin{tabular}{|l|l|l|}
\hline 10 & Sina Weibo & 511 \\
\hline 11 & Telegram & 500 \\
\hline 12 & Snapchat & 498 \\
\hline 13 & Kuaishou & 481 \\
\hline 14 & Pinterest & 4420 \\
\hline 15 & Reddit & 430 \\
\hline 16 & Twitter & 400 \\
\hline 17 & Quora & 353 \\
\hline
\end{tabular}

\section{Related Work}

\subsection{Evaluation of Impact of Image processing on Stego Images}

Each social media network is perceived to have its own image processing method. In 2014, an experiment was conducted by multiple researchers [2] to the find the impact of image processing on certain steganography techniques used by different tools. Since the details of the image processing techniques used by social media websites were not available at the time, the results of the study provided some insight on those methods. In their study, they tested the stego tools GhostHost, Steghide, OutGuess, F5, and Yass on Facebook, Flickr, Google+, and Twitter. Performance of the use of tool and social media was based on if the extracted message matched the original embedded message. Key takeaways from the results are in each subsection below [2].

\subsubsection{Facebook}

Some metadata fields (EXIF data) were removed, and other were found to be overwritten. Since GhostHost uses metadata in its data hiding algorithm, it did not perform well. The researchers also found the usually more than 60 percent of the pixels remained the same, and the other pixel values were modified (with the maximum deviation from the original value being 30 ). The value changes could have been due to JPEG lossy compression, which is speculated to be used by Facebook [2]. Also, image dimensions that are larger the 2048 pixels are resized, which means 
that tools like Steghide, that rely on pixel byte manipulation did not work. It was also found that Facebook used a quality factor of 75 for images most of the time.

\subsubsection{Flickr}

Some metadata fields were removed and other were overwritten like Facebook. Flicker also uses a quality factor of 96 for luminance and chrominance. Flickr and Facebook had very similar image processing making their performance similar as well.

\subsubsection{Google+}

Stego image integrity was preserved by Google + if their dimensions were smaller than $2048 \times 2048$ pixels. All stego tools tested with that constraint were successful.

\subsubsection{Twitter}

Some metadata fields were removed, and others were overwritten like Facebook and Flickr. Also, the stego images was preserved as long as the image was no longer than $1024 \times 768$ pixels.

\subsection{StegHash: New Method for Information Hiding in Open Social Networks}

Researchers proposed a method of information hiding called StegHash (short for Steganographic Hashtags). The method involved a chain of images and videos being posted on different social media with hashtags. Some of the posted images or videos would contain embedded information. The hashtags would be used to connect the different posts. The order of the hashtags for each post affects the ordering of the chain of files when attempting to do the extraction. The success of the method was dependent on the steganography tools that were used when the photos and videos were posted [24] 


\begin{tabular}{|c|c|c|c|c|}
\hline $\begin{array}{l}\text { Hashtags: } \\
\text { \#alpha } \\
\text { \#bravo } \\
\text { \#charlie } \\
\text { Media Type: } \\
\text { Image } \\
\text { Website: } \\
\text { Twitter }\end{array}$ & & $\begin{array}{l}\text { Hashtags: } \\
\text { \#bravo } \\
\text { \#charlie } \\
\text { \#alpha } \\
\text { Media Type: } \\
\text { Image } \\
\text { Website: } \\
\text { Facebook }\end{array}$ & 3 & $\begin{array}{l}\text { Hashtags: } \\
\text { \#bravo } \\
\text { \#alpha } \\
\text { \#charlie } \\
\text { Media Type: } \\
\text { Image } \\
\text { Website: } \\
\text { Instagram }\end{array}$ \\
\hline 1. & 2 & & & \\
\hline $\begin{array}{l}\text { Hashtags: } \\
\text { \#charlie } \\
\text { \#bravo } \\
\text { \#alpha } \\
\text { Media Type: } \\
\text { Image } \\
\text { Website: } \\
\text { Facebook }\end{array}$ & & $\begin{array}{l}\text { Hashtags: } \\
\text { \#bravo } \\
\text { \#charlie } \\
\text { \#alpha } \\
\text { Media Type: } \\
\text { Image } \\
\text { Website: } \\
\text { Twitter }\end{array}$ & 5 & $\begin{array}{l}\text { Hashtags: } \\
\text { \#alpha } \\
\text { \#charlie } \\
\text { \#bravo } \\
\text { Media Type: } \\
\text { Image } \\
\text { Website: } \\
\text { Instagram }\end{array}$ \\
\hline
\end{tabular}

Figure 5: StegHash Method [24]

\subsection{Case Study on Steganography in Social Media}

In 2019, there was a case study conducted by a researcher at Iowa State University on the feasibility of sharing steganographic images to certain social media websites [1]. The researcher also conducted an experiment testing certain tools on multiple social media websites. For their contributions the tools JP Hide \& Seek, and Silent Eye were used on Twitter and Facebook. They also developed a method for uploading steganographic images, which was used in the experiment.

The preliminary steps of the method used in the experiment involved uploading an image to a social media website and downloading the same image. The purpose of the preliminary steps was to create usable cover images for steganography, producing images that have already been compressed by a social media website's image processing algorithm.

The following steps involved creating a stego image with an embedded message using a stego tool, uploading the stego image to a social media website, and then downloading the posted stego image to attempt to extract the embedded message. Table 5 summaries the retrospective case study and the results of their contributions from the experiment. Their contributions from the 
experiment [1] are highlighted in green. The developed method that was used is also shown below in figure 6 .

Table 4: Key Definitions for Table 5 [1]

\begin{tabular}{|c|c|}
\hline Key & Definition \\
\hline N/A & No previous work has been conducted \\
\hline$\checkmark *$ & $\begin{array}{c}\text { During the trials the embedded message was } \\
\text { never retrieved from the downloaded stego } \\
\text { image }\end{array}$ \\
\hline$\checkmark$ & $\begin{array}{c}\text { During the trials the embedded message was } \\
\text { sometimes retrieved from the downloaded } \\
\text { stego image }\end{array}$ \\
\hline & $\begin{array}{c}\text { During the trails using the embedded message } \\
\text { was always retrieved from the downloaded } \\
\text { stego image }\end{array}$ \\
\hline
\end{tabular}

Table 5: Summary of Case Study and Experiment [1]

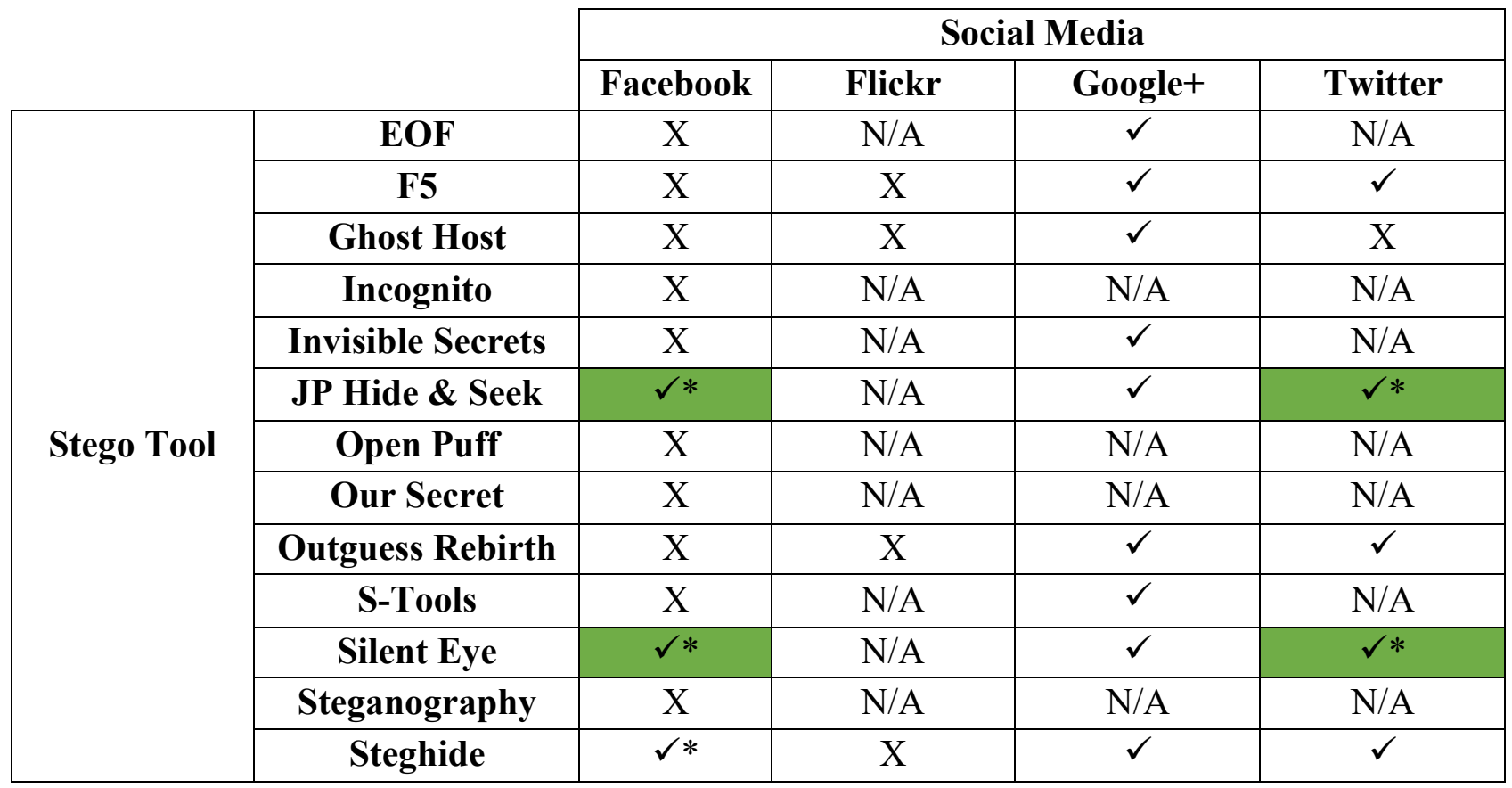




\begin{tabular}{|l|l|l|l|l|l|}
\hline & YASS & $\checkmark^{*}$ & $\checkmark^{*}$ & $\checkmark$ & $\checkmark$ \\
\hline
\end{tabular}

Looking at the results of the case study the most popular social media network, Facebook, did not perform well with most tools. Google+(consumer version no longer active as of April 2019 [3]) and Twitter worked well with most tools that were tested. Flicker, which is a social media network that is based on sharing photos, did not perform well with most tools. The measured performance in all cases were based on if the social media websites preserved the integrity of a stego image or not.

Step 1

\begin{tabular}{|l|}
\hline Take \\
picture \\
with phone
\end{tabular}

Step 2

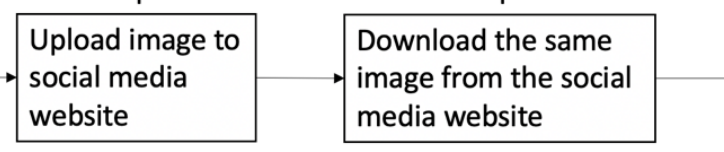

Step 6

Download the same stego image and then attempt to extract that embedded message using a stego tool
Step 4

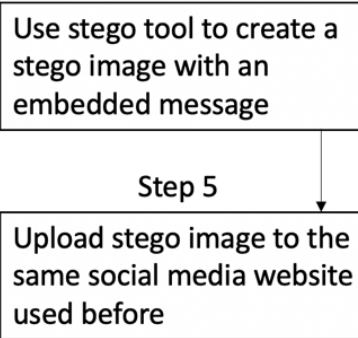

Step 5

Upload stego image to the same social media website used before

Figure 6: Developed Method used in Experiment

\subsection{Contributions}

For my contributions, an experiment was conducted using DIIT, Steghide, OutGuess, and ExifTool on Twitter and Reddit. In the study conducted by Trotter [1], Reddit was never tested with any tool. DIIT, OutGuess (non-rebirth), and ExifTool were also not tested with any social media website. Also, since the work conducted by researchers [2] was in 2014, the combination of Twitter and Steghide needed to be revisited for the possibility of Twitter updating its image processing algorithm.

\section{Problem Statement}

As mentioned before, social media websites appear to be an attractive medium for sharing steganographic images because the volume of user activity, the interconnectivity that it provides, and rarity of steganographic images being posted. Each social media website is also assumed to have their own image processing algorithms. Since there isn't any publicly available information 
or documentation on each algorithm, certain steganographic techniques and social media combinations have to be verified to test the feasibility of using social media for this method of secret communication.

\section{Description of Pilot Experiment}

For the pilot experiment, one cover image was used to create 320 stego images using the tools DIIT, Steghide, OutGuess, and ExifTool (40 images per stego tool and social media combination). The social media websites that were used were Twitter and Reddit. The cover image was posted on each social media website and then downloaded. The downloaded image from each website was then used to create a stego image that would contain a text file with a message. The stego image was then uploaded to the same social media website used before and then downloaded again. After downloading the posted stego image an attempt was made to extract the message using the same stego tool that created it. This process was repeated 40 times for each combination of stego tool and social media website. The purpose of the pilot experiment was to measure the reliability of a modified version of a method developed in a previous study [1]. This made sure that if the exact method were repeated the exact result would occur each time. The results of the pilot statement proved the method to be reliable, being that the results were consistent.

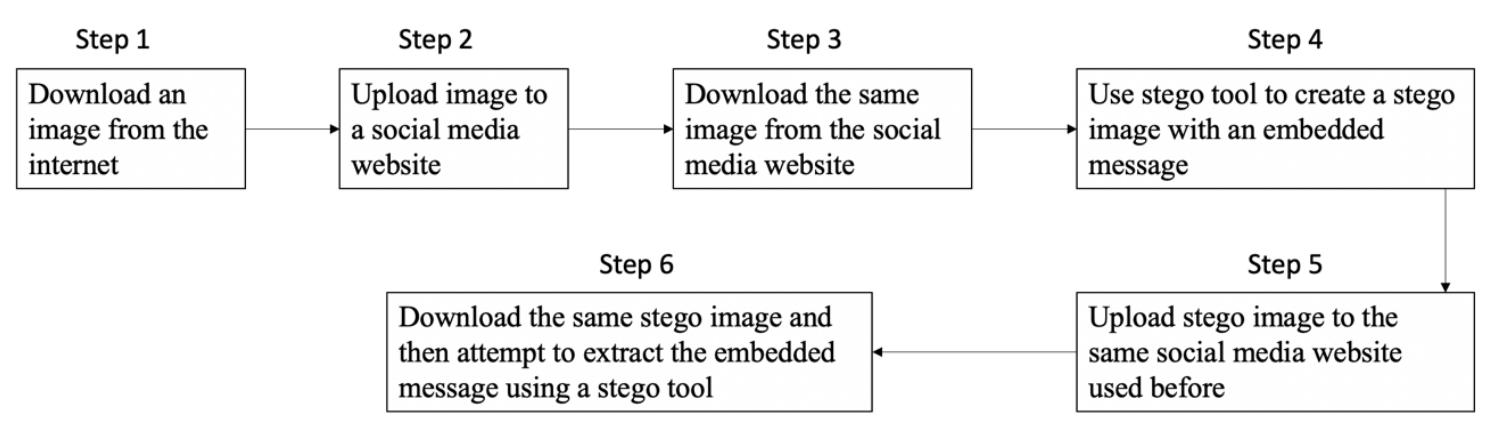

Figure 7:Modified Method for Posting Steganographic Images to Social Media [1]

\section{Description of Experiment}


To continue the research that was conducted by [1], more combinations of stego tools and social media websites were tested using the method described in Section 6.1. The tools DIIT, Steghide, Outguess, and ExifTool were tested with Twitter and Reddit. The details of each tool are discussed in Section 6.1. Each combination of stego tool and social media are shown in Table 6. Twitter and Reddit were used because they both rank in the top 20 most popular social media websites in the world [4], and they are both used for communication and sharing media. They also allow users to downloaded posted image without screenshotting. Also, Reddit has not been tested in the past according the case study conducted [1].

Since the most popular social media website, Facebook, was studied heavily in the past, it was not chosen for this reason. YouTube and Tik Tok were not chosen since they mostly used to post videos and not images. Instagram, which is another very popular social media website, was not choses since it does not give users the ability to download posted images without screenshotting. The tools DIIT, Steghide, and OutGuess, and ExifTool were chosen because there were free and were either compatible with Linux, MacOS, or Windows operating systems. The links for download of each tool used are in Table 6.

Table 6: Stego Tool Links

\begin{tabular}{|l|l|}
\hline Stego Tool & Link \\
\hline DIIT(BattleSteg) & http://diit.sourceforge.net/ \\
\hline Steghide & http://steghide.sourceforge.net/ \\
\hline OutGuess & https://manpages.ubuntu.com/manpages/xenial/man1/outguess.1.html \\
\hline ExifTool & https://exiftool.org/ \\
\hline
\end{tabular}

\subsection{Method}

A modified version of the method developed in a pervious study [1] was used because in the original method only images of one size were tested (all images produced in step 1 of the original method were by one camera on a phone). The modified method replaces step 1, downloading images directly from Google, which allows the rest of the method to be tested with different image sizes. 
The method used in this experiment and pilot experiment was selected because it was recently developed, and it also had some success during previous testing [1]. As mentioned before, the method was only tested with four combinations of a stego tool and social media.

In the experiment, one trial consisted of testing one stego tool and social media combination with different images. In each trial, steps 3,4, and 5 all produced an image. For step 1, images of random sizes were downloaded from Google images using the search word "random". Instead of repeating steps 1,2, and 3 for each trial, the images that were downloaded, uploaded, and redownloaded to the social media websites in the first trial were reused in the other trials for consistency. In step 4, all images were embedded with the same text file. In step 5, the same computer used to create the stego image was used in the attempt to extract the embedded message. All steps were completed on either on a MacOS or Linux computer and only one network was used. 40 images with dimensions ranging from 200x200 to 3500x1969 were used in each trial. In total, 720 images were produced in the process of conducting trails.

\subsubsection{Experiment Details}

The experiment involved two factors with one having four levels and the other having two levels. The stego tool was one factor and the social media was the other factor. The success rate of extracting a message from an image, was dependent on the combination of the two.

Table 7: All Combinations for the Experiment

\begin{tabular}{|c|c|c|c|}
\cline { 3 - 3 } \multicolumn{2}{c|}{} & \multicolumn{2}{c|}{ Social Media } \\
\cline { 2 - 4 } \multicolumn{2}{c|}{} & Twitter & Reddit \\
\hline \multirow{3}{*}{$\begin{array}{c}\text { Stego } \\
\text { Tool }\end{array}$} & DIIT(BattleSteg) & $\mathrm{C}_{1}$ & $\mathrm{C}_{2}$ \\
\cline { 2 - 4 } & Steghide & $\mathrm{C}_{3}$ & $\mathrm{C}_{4}$ \\
\cline { 2 - 4 } & OutGuess & $\mathrm{C}_{5}$ & $\mathrm{C}_{6}$ \\
\cline { 2 - 4 } & ExifTool & $\mathrm{C}_{7}$ & $\mathrm{C}_{8}$ \\
\hline
\end{tabular}

$\mathrm{C}_{1}$ : Digital Invisible Ink Toolkit and Twitter 
$\mathrm{C}_{2}$ : Digital Invisible Ink Toolkit and Reddit

$\mathrm{C}_{3}$ : Steghide and Twitter

$\mathrm{C}_{4}$ : Steghide and Reddit

$\mathrm{C}_{5}$ : OutGuess and Twitter

$\mathrm{C}_{6}$ : OutGuess and Reddit

$\mathrm{C}_{7}$ : ExifTool and Twitter

$\mathrm{C}_{8}$ : ExifTool and Reddit

\subsubsection{Digital Invisible Ink Toolkit (DIIT)}

The Digital Invisible Ink Toolkit is a free stego tool that can encode and decode steganographic images. It provides a graphic user interface for its users it is and it compatible with any OS that has a Java Runtime Environment with it being a JAR File (Java Archive). Figures 8 and 9 show the encoding and decoding windows for the tool. When using the encode feature (pictured in figure 8), the user can select the cover image, the steganography algorithm and the text file which will be embedded, and the location where the location where the stego file will be saved. The user can also further protect the stego image from extraction by adding a password. When the decode feature is used (pictured in figure 9), the user can select the stego image, choose the algorithm, and the file location where they want to save the extracted data. The user will also have to enter the password if one was used during the encoding process.

In the trials conducted, version 1.5 of DIIT was used. The Battlesteg algorithm was used with its default settings. In the trials conducted it was the only tool that had a graphic user interface. The Battlesteg algorithm uses a filter to find the highest values labeling them as "ships". The options for the filter include Laplace, Sobel, and Prewitt. The algorithm then randomly "shoots" at the image in hopes of finding a "ship". Once a ship is found, the following "shots" are then grouped around the found "ship" until it "sinks" or it is completely covered. The process is repeated until all the data is embedded. When using the tool, the user can specify the range of the "shots" and the maximum number of "shots" on a found "ship". For the trials the default settings were used and stego images were saved as PNGs. 


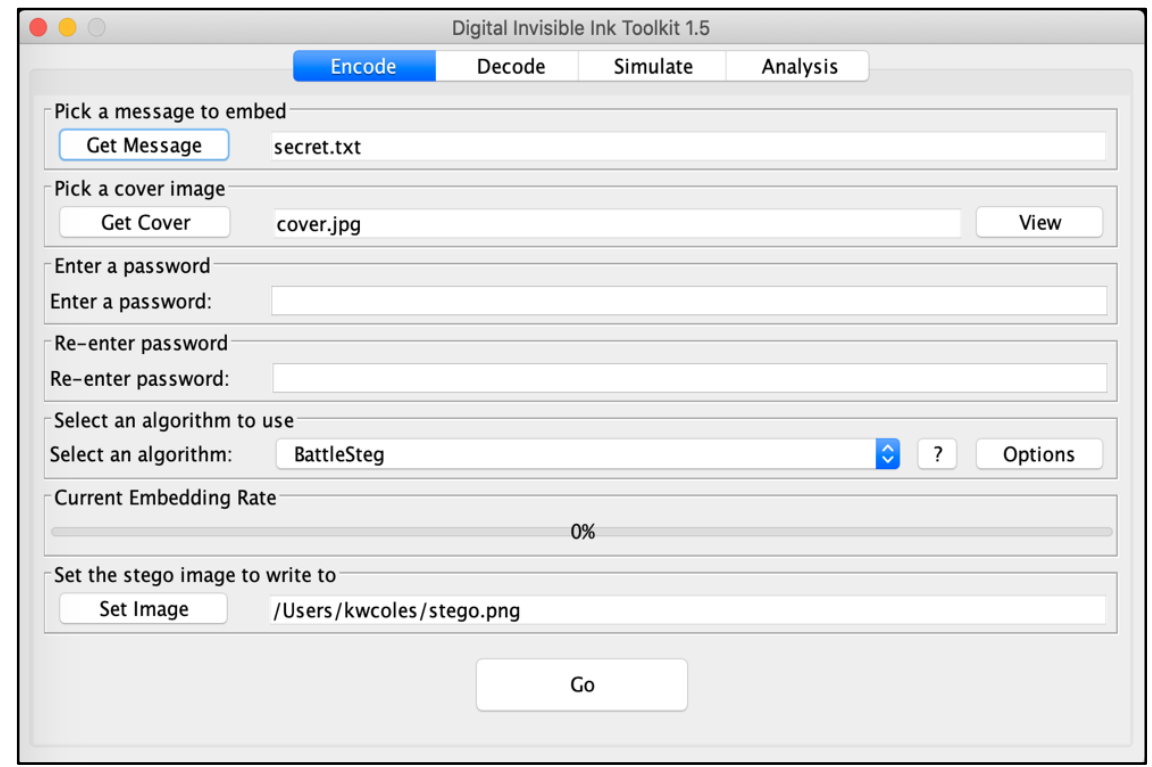

Figure 8: Encode Window

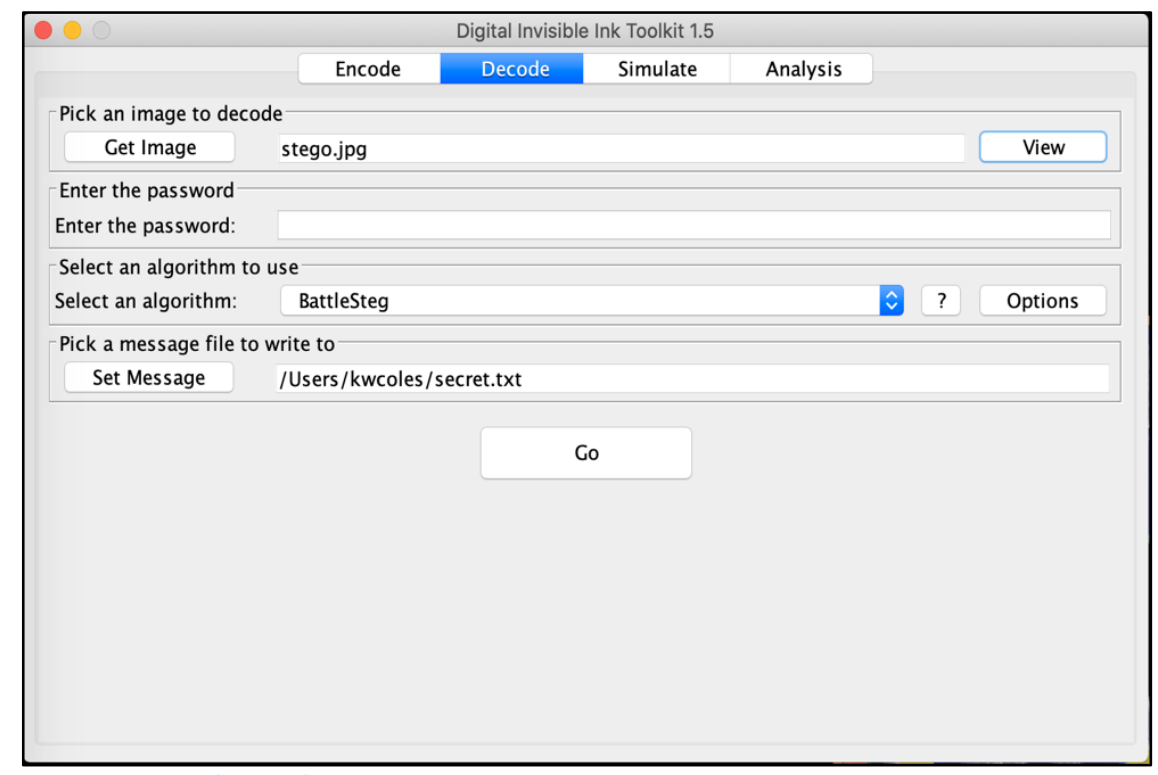

Figure 9: Decode Window

\subsubsection{Steghide}

Steghide [6] is another free stego tool that has the ability encoding and decoding steganographic images. It does not provide a graphical user interface as it can only be used on the command line. It's also only compatible with Windows and Linux operating systems. Figure 9 shows an example of how to create a stego image and extract an embedded message from an image. 
In the embed command, the "-cf" flag is for the cover file, and the "-ef" flag is for the file that is going to be embedded. After running this command, the users are prompted to enter a password, which further protects the message from being extracted. For the extract command, the "-sf" flag is for the stego file. After running this command users are prompted to enter the passphrase in order to extract the message. If the correct passphrase is entered, the embedded file is saved to the current directory that the tool is running in. In figure, which shows the example, the extracted text file, named "secret.txt" is saved to the Desktop directory.

During the embedding process, the embedded file is compressed and then encrypted with Rijndael with a key size of 128 bits (Advanced Encryption Standard). A checksum is also embedded using CRC32 (Cyclic Redundancy Check) to verify the integrity of the embedded file during the extraction process. The embedding algorithm itself, gets a sequence on pixels from the cover file based on a pseudo random number created from the passphrase. The sequence of numbers corresponds with the position of where the secret data will be embedded. There are some cases where the pixel at a particular position does not need to be changed. When this occurs, the exact positions are noted. Then graph theory is used to find pairs such that exchanging's their values do not have an effect on the part of the secret data. The corresponding pixels of the remaining positions that could not find pairs are modified. Most of the embedding is done by exchanging pairs. [7]

For the trials in the experiment, Steghide version 0.1.5 was used to create the stego images. The exact commands pictured in figure 10 were used create stego images and extract the embedded text file, meaning that secret text was compressed and encrypted with AES. A checksum was used as well. No passwords were used to protect the stego image. The JPEG image format was used for the cover file and stego image [7].

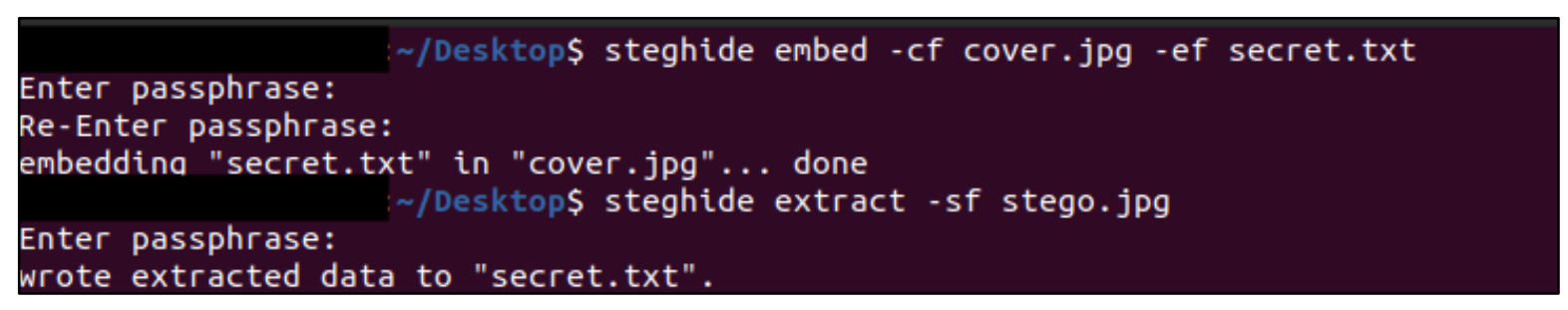

Figure 10: Steghide Example 


\subsubsection{OutGuess}

Outguess is a another free steganographic tool used for encoding and decoding stego images. Figure 11 shows an example of how to use the tool which compatible with Windows and Linux operating systems. It also can be only used with the command line. The embedding example is shown in figure 11. In the embed command the "-d" flag is for the datafile that is being embedded, which in this case is the "secret.txt" file. The datafile in the command is followed by the cover image and the output image that the data is embedded in. Users also have the option to add a password. The extraction example is also shown in figure 11. In the extract command the "-r" flag is to signal to retrieve a message from a stego file. The first file listed after the flag is the stego file and the second file listed in the command the name of the destination file that will be overwritten with the extracted data. One unique feature of the OutGuess tool is that it has the ability to hiding multiple messages in a cover image. In the embedding process the tool uses an iterator to find the best bits to hide data [24].

For the trials in the experiment, exact commands pictured in figure 11 were used to create stego images and to extract data from them. No password was used to further protect the data.

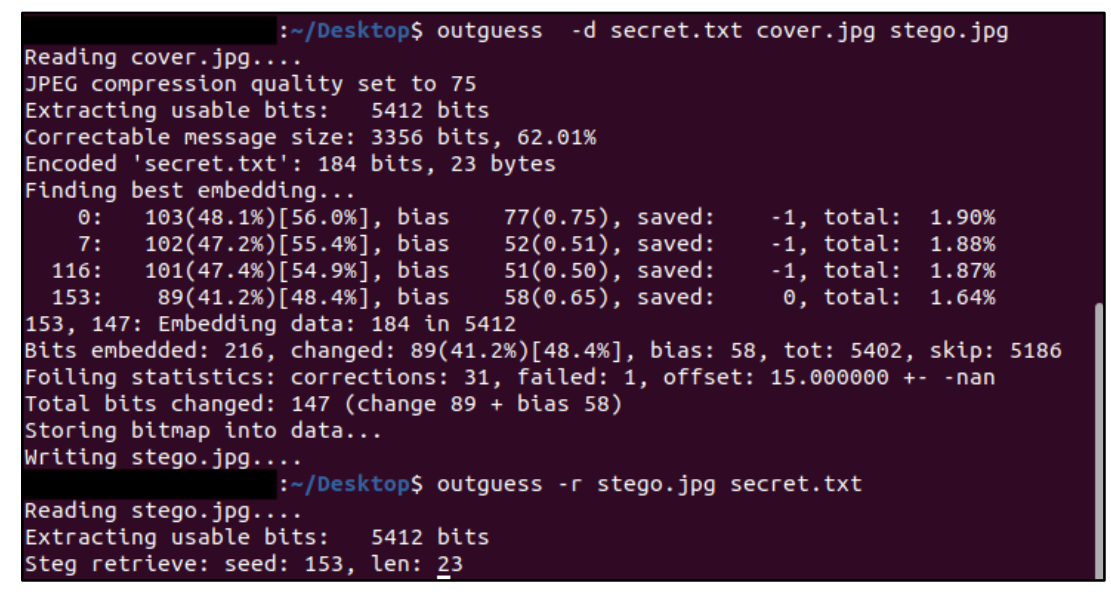

Figure 11: OutGuess Example

\subsubsection{ExifTool}

ExifTool [27] is a free tool that can be used to read, write and edit metadata or EXIF data to many different file types. It is compatible with Windows, MacOS, and Linux operating systems. Figure 12 shows an example of how to use the tool to edit an image's metadata. In the example a tag named "comment" is added to the cover image. The same figure also shows how to extract an 
image's metadata. The "-v" flag stands for verbose, meaning that more metadata is shown than in the default configuration.

For the trials in the experiment, a tag was added to the images using this method described above and pictured in figure 12 .

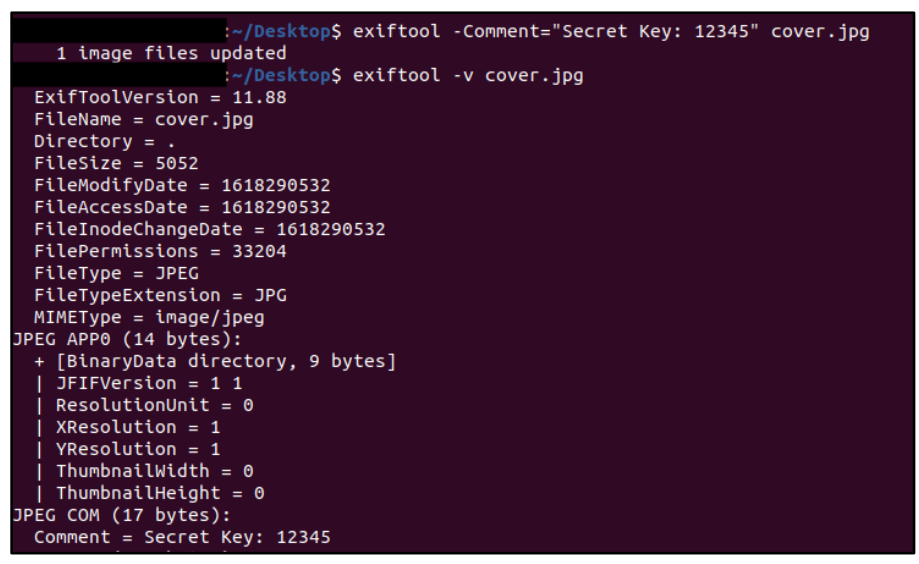

Figure 12: ExifTool Example

\section{Results and Interpretation}

\subsection{Image Dataset}

Most of the images that were downloaded in step 1 were below $500 \mathrm{~KB}$ in file size. All the images were also downloaded as JPEGs. The dimensions of the images ranged from 200x200 to 3500x1969. These images also varied in quality. 


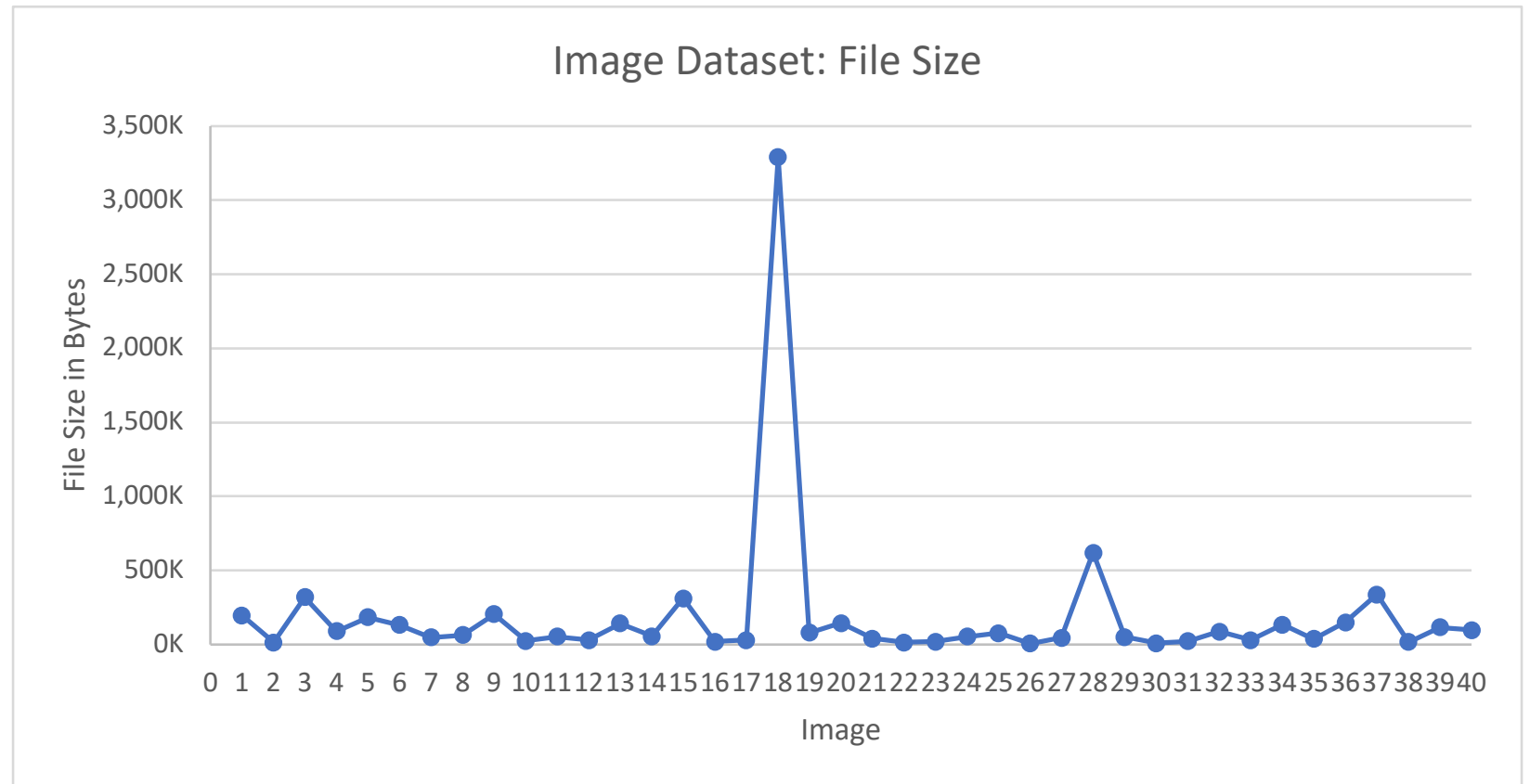

Figure 13: Image Dataset produced from Step 1 of the Method

\subsection{Trial Results}

For the results of the trials conducted, the successes were recorded when the embedded message was retrieved. Failures were recorded when the embedded message was not retrieved. The success rate was calculated by the total amount of successes divided by the total amount of images posted. Table 8 summarizes the results of the entire experiment and it also used the same key described in table 4.

\begin{tabular}{|c|c|c|c|}
\hline & & \multicolumn{2}{|c|}{ Social Media } \\
\hline & & Twitter & Reddit \\
\hline \multirow{4}{*}{ Stego Tool } & DIIT(BattleSteg) & $\checkmark *$ & $\checkmark$ \\
\hline & Steghide & $\checkmark$ & $\checkmark$ \\
\hline & OutGuess & $X$ & $\checkmark$ \\
\hline & ExifTool & $\mathrm{X}$ & $\checkmark$ \\
\hline
\end{tabular}




\subsubsection{Twitter}

Twitter, which has been used in the past, has shown to be a decent medium to share steganographic images. In the previous study [1] it has shown being an effective medium when using F5, JP Hide \& Seek, OutGuess Rebirth, Steghide, and YASS as tools. In the experiment, the DIIT and Twitter combination had success since the embedded message being extracted more than half of the time, but it still not considered to be reliable. When retesting the Steghide tool, it still proved to reliable, as it did the past [2]. When testing the OutGuess and ExifTool on twitter, they weren't any recorded successes in extracting the embedded message.

Table 9: Results for All Twitter Images

\begin{tabular}{|c|c|c|c|}
\hline Combination & Successes & Failures & Success Rate \\
\hline $\begin{array}{c}\mathrm{C}_{1} \text { : Digital Invisible Ink } \\
\text { Toolkit and Twitter }\end{array}$ & 25 & 15 & $25 / 40=62.50 \%$ \\
\hline $\mathrm{C}_{3}$ : Steghide and Twitter & 40 & 0 & $40 / 40=100.00 \%$ \\
\hline $\mathrm{C}_{5}$ : OutGuess and Twitter & 0 & 40 & $0 / 40=0.00 \%$ \\
\hline $\mathrm{C}_{7}$ : ExifTool and Twitter & 0 & 40 & $0 / 40=0.00 \%$ \\
\hline
\end{tabular}

When considering the recommended image size threshold (1024x768) for preserving an image's integrity on twitter [2], only one combination, being $\mathrm{C}_{1}$, improved in performance. When the images were above the recommended threshold, there weren't any recorded successes in using $\mathrm{C}_{1}$. The improvement in success rates for one of the combinations show that the threshold appears to be a good estimate for the tools that have shown any success of being usable.

Table 10: Twitter Results Below Threshold

\begin{tabular}{|c|c|c|c|}
\hline Combination & Successes & Failures & Success Rate \\
\hline $\begin{array}{c}\mathrm{C}_{1} \text { : Digital Invisible Ink } \\
\text { Toolkit and Twitter }\end{array}$ & 25 & 5 & $25 / 30=83.33 \%$ \\
\hline $\mathrm{C}_{3}$ : Steghide and Twitter & 30 & 0 & $30 / 30=100.00 \%$ \\
\hline
\end{tabular}




\begin{tabular}{|c|c|c|c|}
\hline C $_{5}$ : OutGuess and Twitter & 0 & 30 & $0 / 30=0.00 \%$ \\
\hline C $_{7}$ : ExifTool and Twitter & 0 & 30 & $0 / 30=0.00 \%$ \\
\hline
\end{tabular}

Table 11: Twitter Results Above Threshold

\begin{tabular}{|c|c|c|c|}
\hline Combination & Successes & Failures & Success Rate \\
\hline $\begin{array}{c}\mathrm{C}_{1} \text { : Digital Invisible Ink } \\
\text { Toolkit and Twitter }\end{array}$ & 0 & 10 & $0 / 30=0.00 \%$ \\
\hline $\mathrm{C}_{3}$ : Steghide and Twitter & 10 & 0 & $10 / 10=100.00 \%$ \\
\hline $\mathrm{C}_{5}$ : OutGuess and Twitter & 0 & 10 & $0 / 30=0.00 \%$ \\
\hline $\mathrm{C}_{7}:$ ExifTool and Twitter & 0 & 10 & $0 / 30=0.00 \%$ \\
\hline
\end{tabular}

\subsubsection{Reddit}

After testing all of the combinations, Reddit seemed to be a great website for posting steganographic images. Regardless of the tool or steganography technique that was used or the size of the image or format of the image, the embedded message was always retained.

Table 12: Reddit Results for All Images

\begin{tabular}{|c|c|c|c|}
\hline Combination & Successes & Failures & Success Rate \\
\hline $\begin{array}{c}\mathrm{C}_{2}: \text { Digital Invisible Ink } \\
\text { Toolkit and Reddit }\end{array}$ & 40 & 0 & $40 / 40=100.00 \%$ \\
\hline $\mathrm{C}_{4}$ : Steghide and Reddit & 40 & 0 & $40 / 40=100.00 \%$ \\
\hline $\mathrm{C}_{6}:$ OutGuess and Reddit & 40 & 0 & $40 / 40=100.00 \%$ \\
\hline $\mathrm{C}_{8}:$ ExifTool and Reddit & 40 & 0 & $40 / 40=100.00 \%$ \\
\hline
\end{tabular}


\begin{tabular}{|l|l|l|l|}
\hline & & \\
\hline
\end{tabular}

\subsubsection{Key Takeaways and Updated Table}

Tools that used spatial domain technique had good success rates with Twitter and Reddit (DIIT and Steghide). When considering the threshold of $1024 \times 768$, Twitter's performance increased. Reddit had a perfect success rate regardless of the image steganography technique that used.

Table 5 combines the work completed in a previous case study, with the results of the experiment on Twitter and Reddit highlighted in blue. Reddit seems to look like a promising medium for sharing stego images, as Google+ has looked in the past (no longer active).

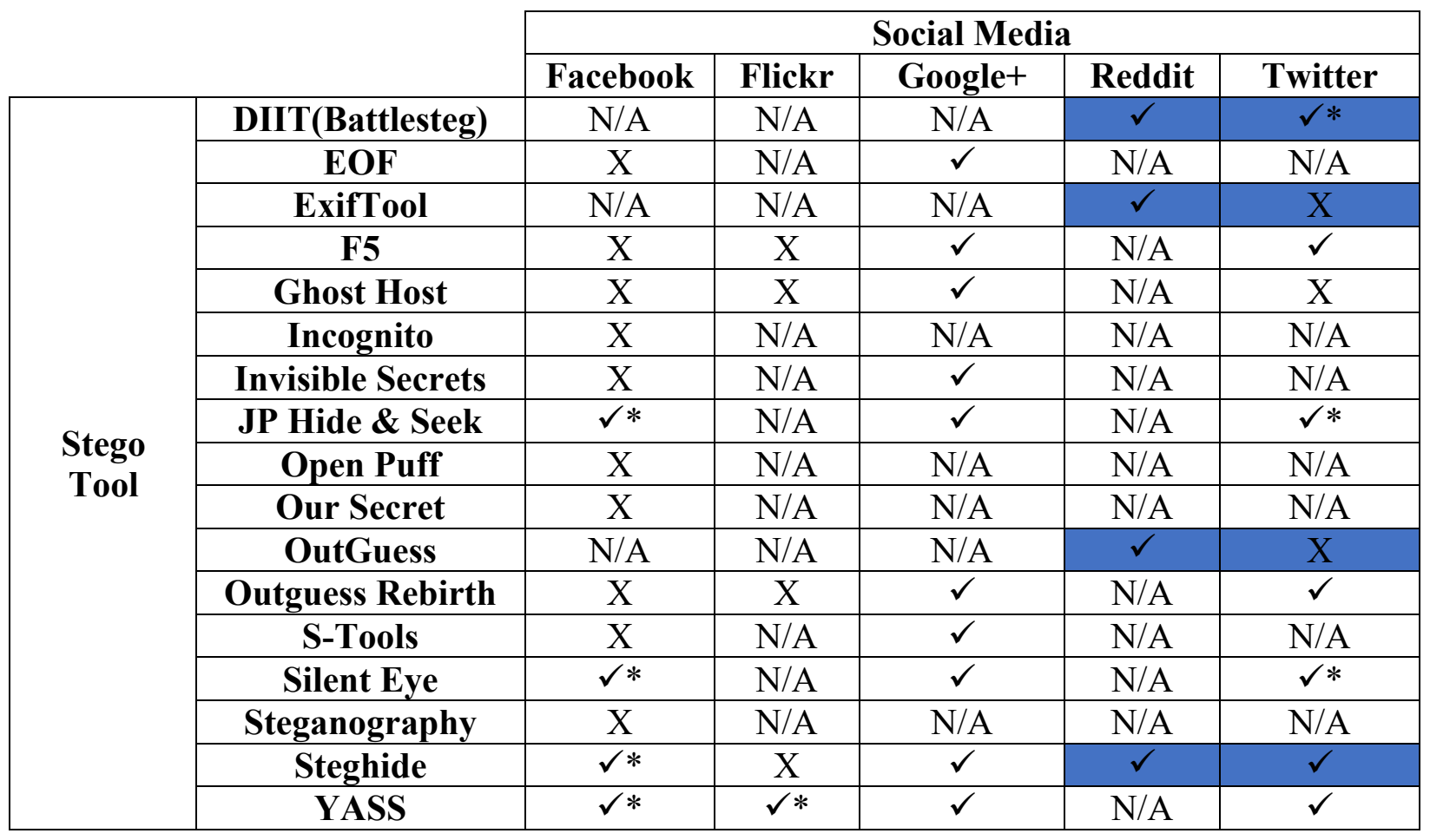

\subsection{Compression Ratios}


Compression ratios were calculated by dividing the uploaded image's size by the corresponding downloaded image's size. A ratio less than 1 implies that the image's file size was decreased.

\subsubsection{Twitter}

When comparing the sizes of non stego images that were uploaded and downloaded from Twitter, it reveals that Twitter image compressing algorithm usually reduces an image size. There were a couple outliers where the image increased in file size. This could be due to Twitter adding metadata fields to images after they were uploaded [2].

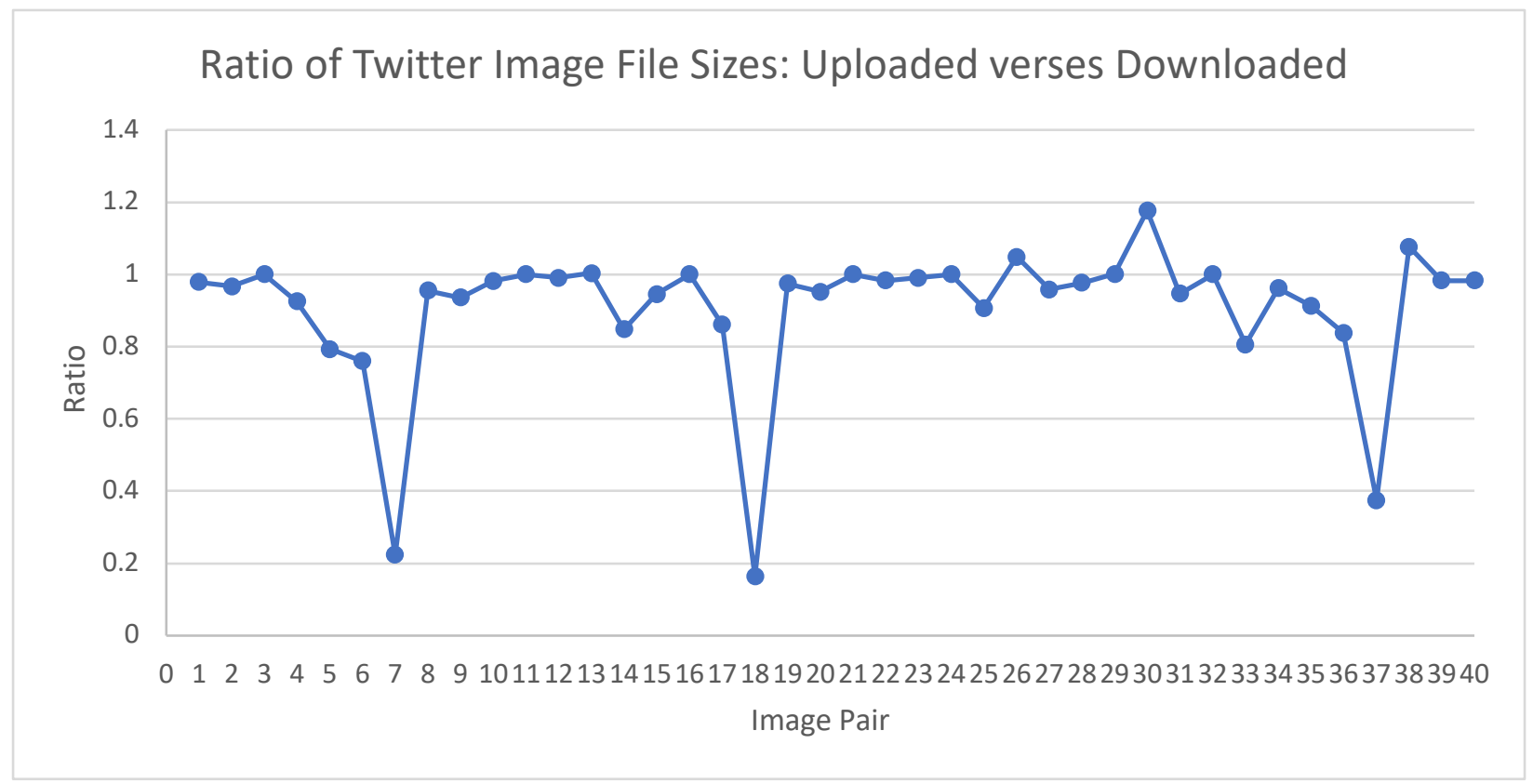

Figure 14: Compression Ratios of Twitter Images

\subsubsection{Reddit}

When comparing the sizes of non stego images that were uploaded and downloaded from Reddit, it revealed that the Reddit image processing algorithm usually does not reduce an image's file 
size (shown in figure 15). Most of the images uploaded maintained the same size when downloaded.

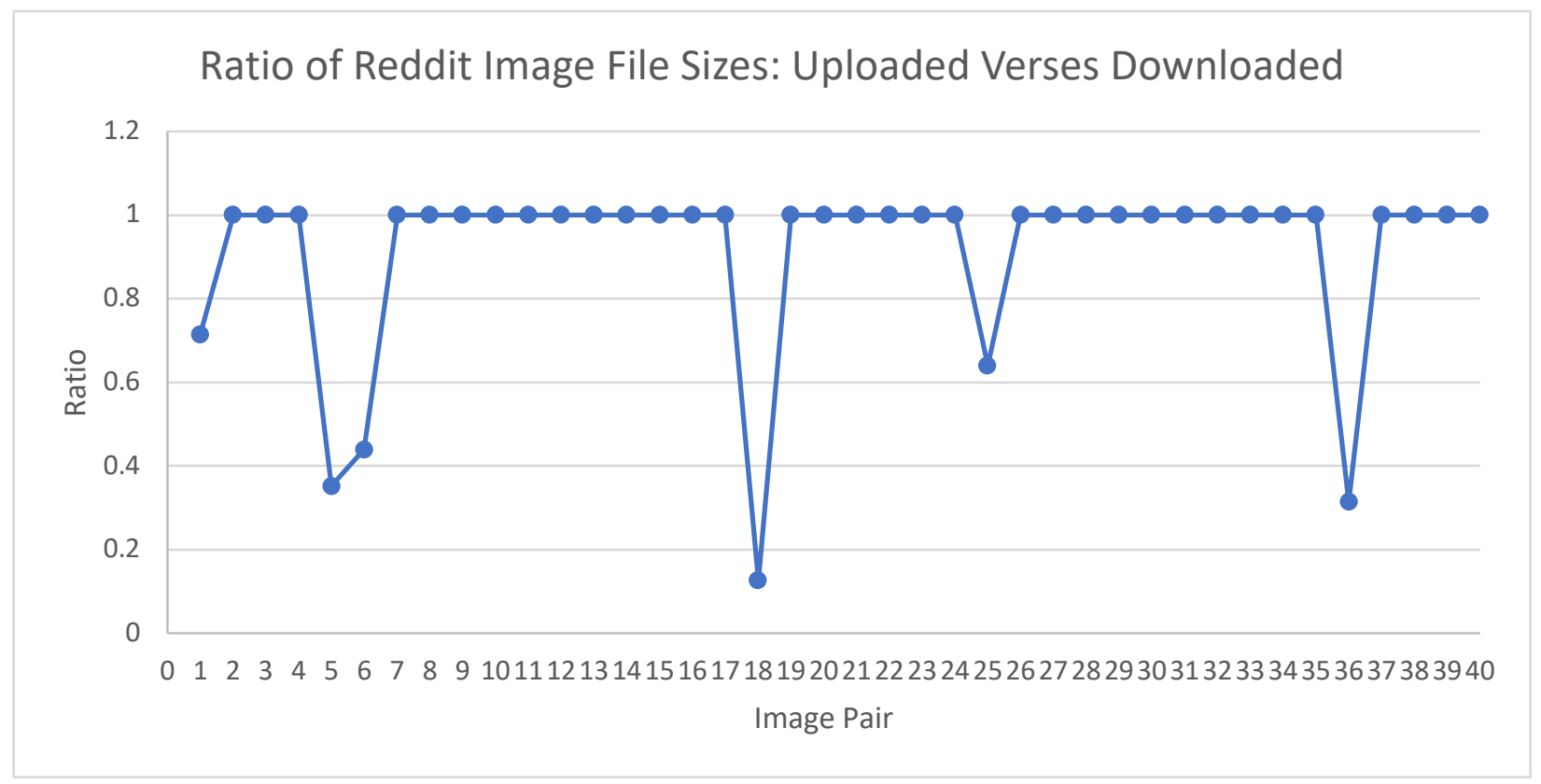

Figure 15: Compression Ratios of Reddit Images

\subsubsection{Key Takeaways}

Twitter usually reduced an uploaded image's file size. Reddit on the other hand, usually preserved an uploaded image's file size.

\subsection{MSE of Uploaded and Downloaded Images}

Mean Square Error (MSE) compares two images $\mathrm{x}_{1}$ and $\mathrm{x}_{2}$ pixel by pixel, to measure the difference between the two. The MSE function accomplishes this by doing a sum of the squared differences between pixel values of two images. The lower the MSE score, the less amount of difference between the two images (less amount of added noise or different pixel values). If the MSE score is zero, then the images are the same pixel wise.

For each website, MSE was calculated between uploaded and downloaded images for non stego and stego images created by each tool. The equation pictured in figure 16 was used to calculate the MSE scores. The reason for calculating the MSE for each image pair (one pair consists of an 
uploaded image and corresponding downloaded image) was to see if there was a pattern in the scores that could relate to the performance of Twitter and Reddit in the experiments.

$$
\mathrm{MSE}=\frac{1}{m n} \sum_{i=0}^{m-1} \sum_{j=0}^{n-1}[I(i, j)-K(i, j)]^{2}
$$

Figure 16: MSE Equation

\subsubsection{Twitter and Reddit}
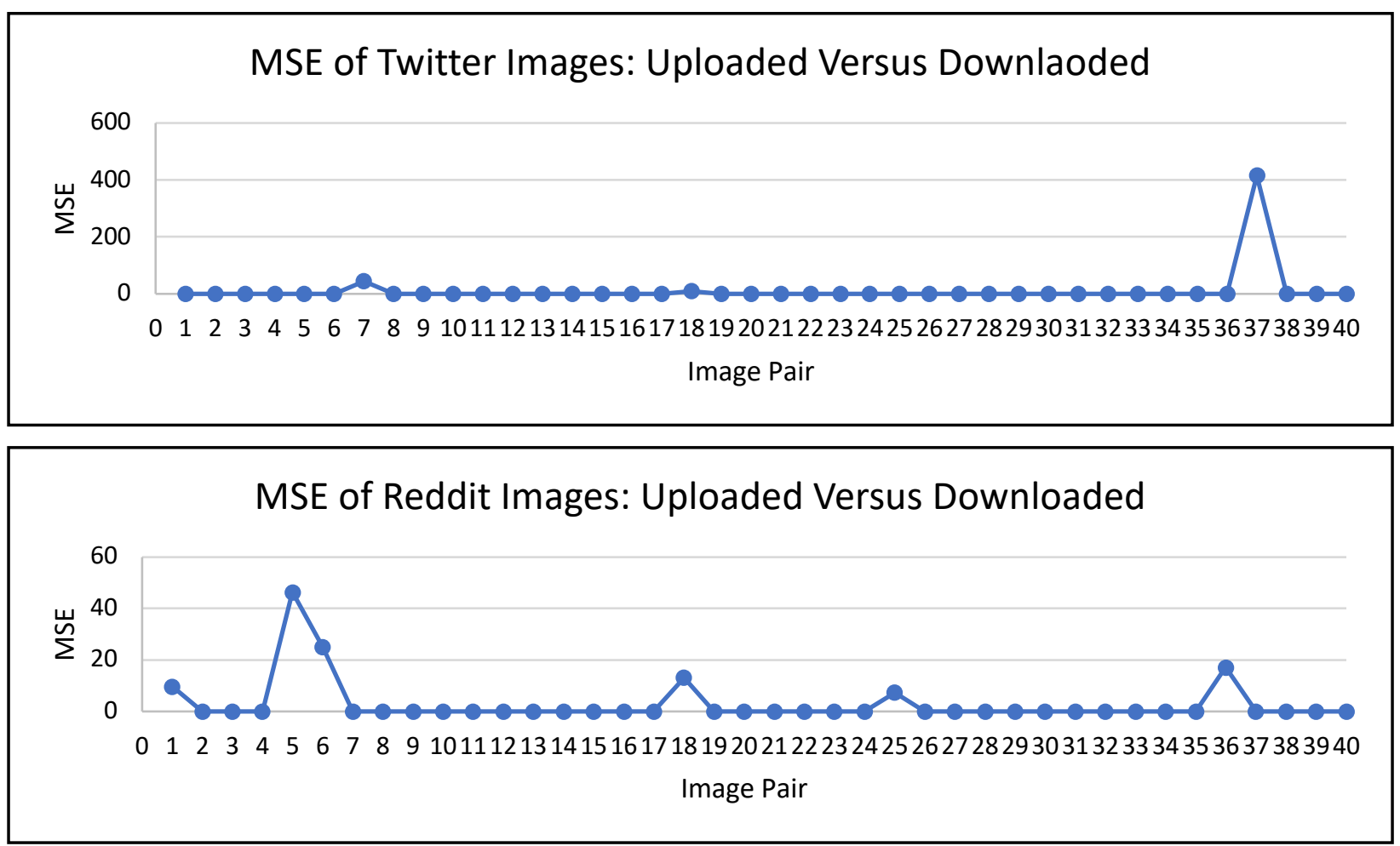

Figure 17: MSE of Twitter and Reddit Images 

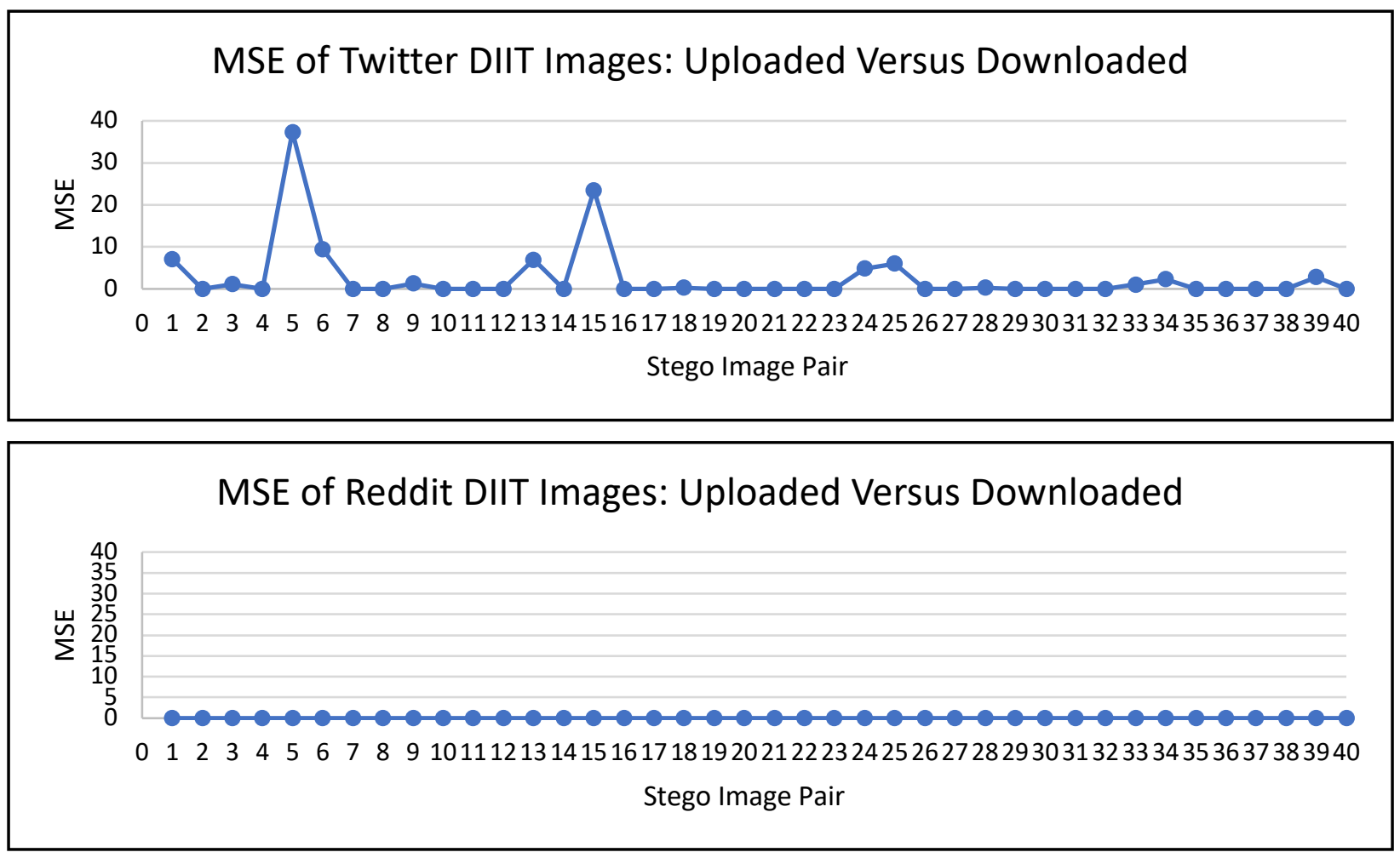

Figure 18: MSE of Twitter and Reddit DIIT Images
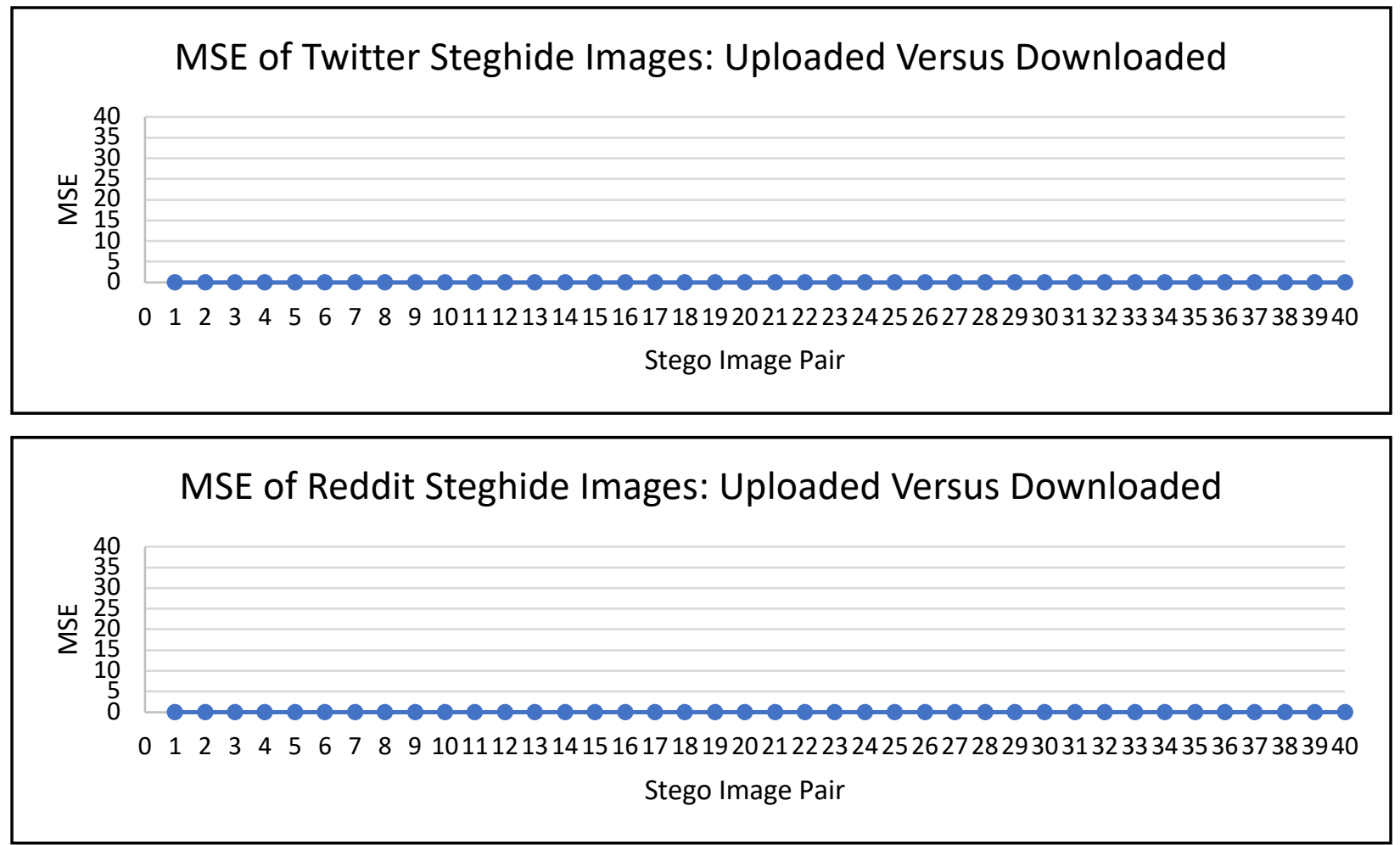

Figure 19: MSE of Twitter and Reddit Steghide Images 

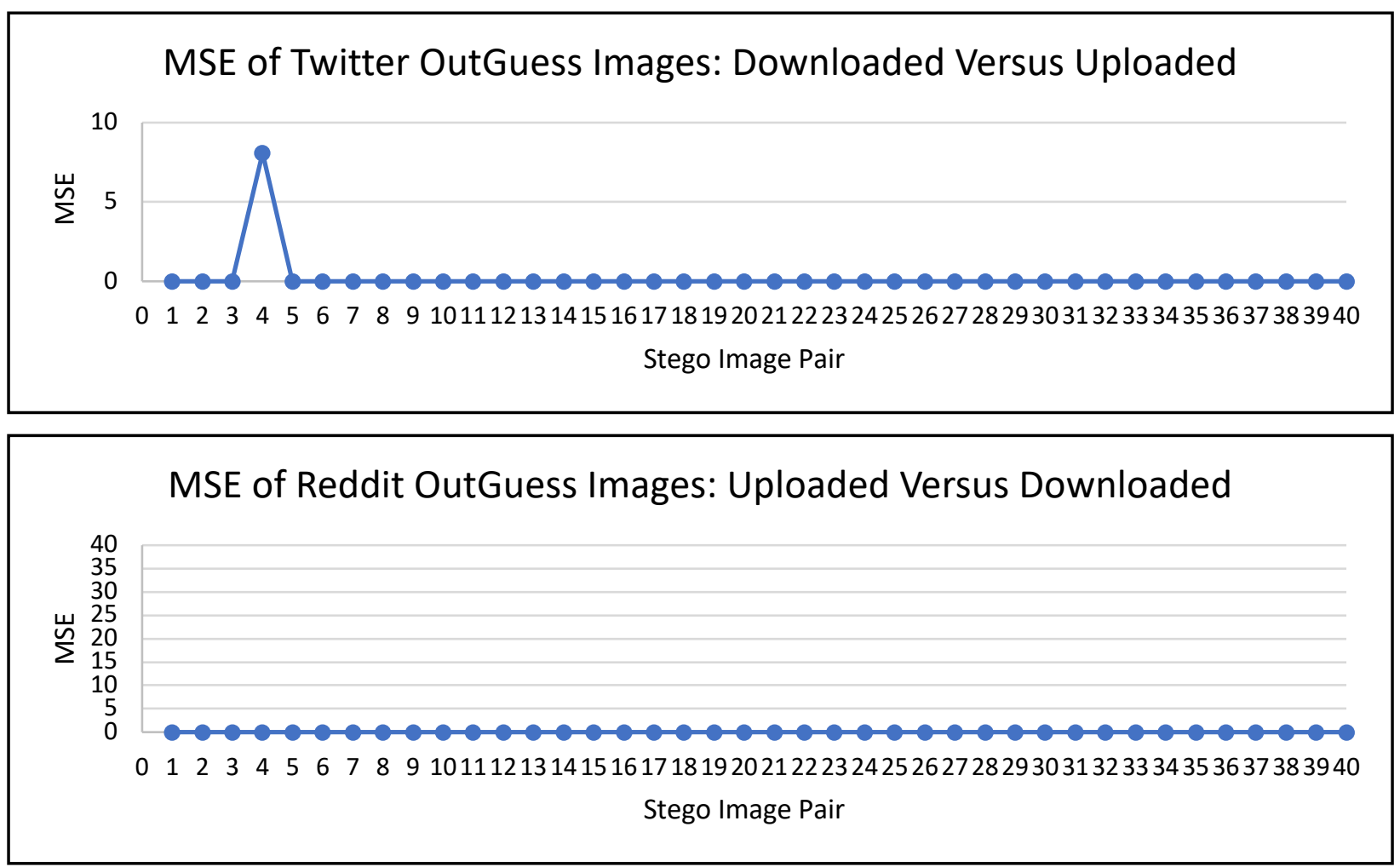

Figure 20: MSE of Twitter and Reddit OutGuess Images
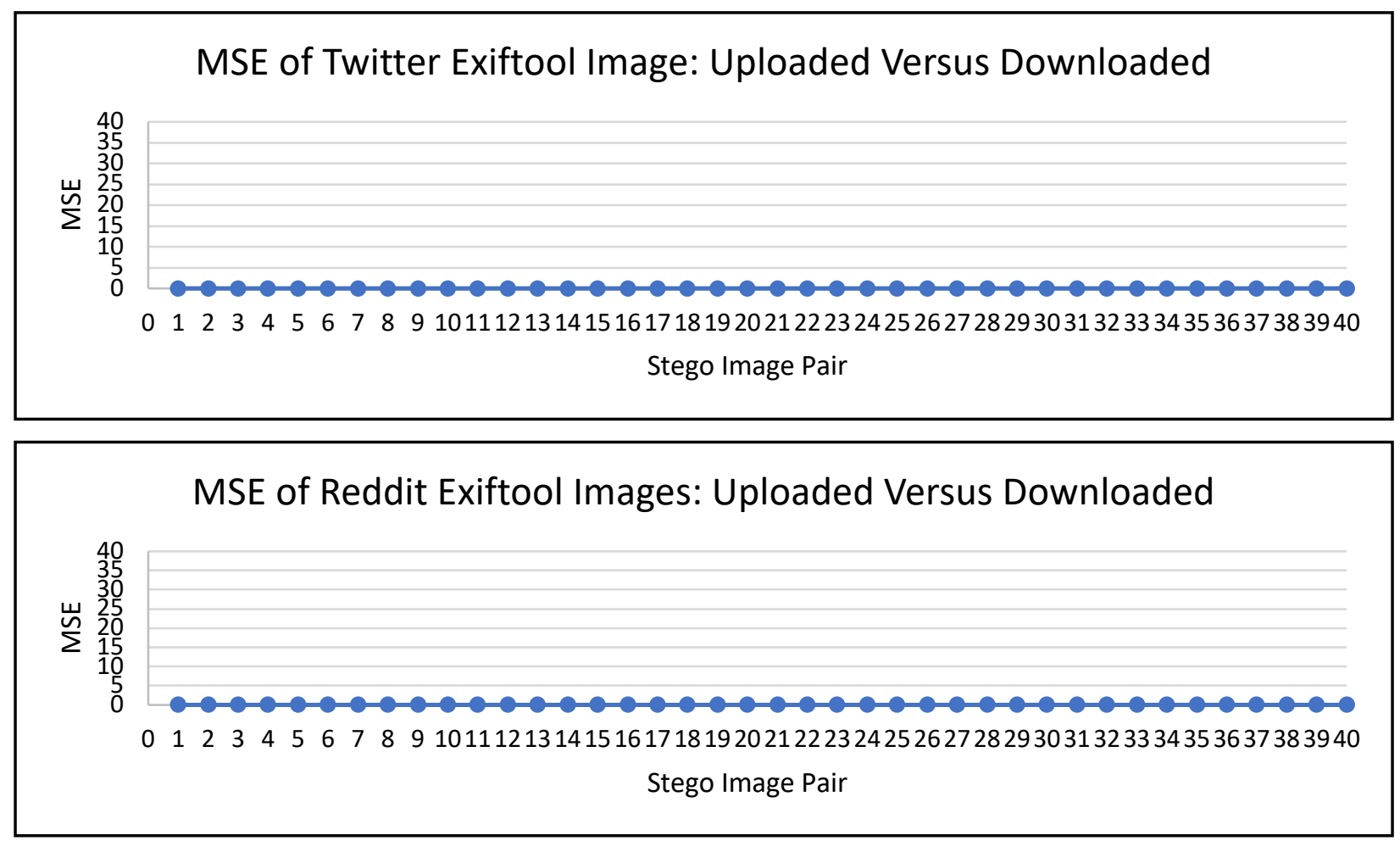

Figure 21: MSE of Twitter and Reddit ExifTool Images 


\subsubsection{Key Takeaways}

When calculating the Mean Squared Error (MSE) of non stego and stego images (uploaded versus downloaded) there wasn't a clear pattern that related to the performance of a particular combination of stego tool and social media website. For example, Steghide had zero MSE for all image pairs (one image pair is uploaded and downloaded image) and it was shown to have great success in its performance in the experiment. OutGuess and ExifTool had a similar MSE charts and both tools had poor success rates with Twitter. DIIT had the most sporadic MSE chart and it performed better than Outguess and Exiftool on Twitter.

\subsection{Quantization Tables}

Former research has shown that some stego tools produce the same quantization tables for every stego image that it creates [1][21]. This could help identify which tool or algorithm is being used when trying to extract embedded information from a stego image. They also can be used to identify which program or type of camera produced the exact image [21].

For the experiment, a digital forensics tool (https://29a.ch/photo-forensics/\#jpeg-data ) was used to get the quality factor of all uploaded and downloaded non-stego and stego images. The values for uploaded and downloaded images were compared to see if the social media websites or tools produced images with a particular quality factor.

\subsubsection{Original Images Downloaded in Step 1}

The quality factors for the images that were downloaded in step 1 are listed in table 14 .

Table 14: Quality Factor Frequency of Original Images

\begin{tabular}{|l|l|}
\hline $\begin{array}{l}\text { Quality } \\
\text { Factor }\end{array}$ & Frequency \\
\hline 80 & 10 \\
\hline 90 & 5 \\
\hline 92 & 4 \\
\hline 75 & 4 \\
\hline
\end{tabular}




\begin{tabular}{|l|l|}
\hline 85 & 3 \\
\hline 93 & 2 \\
\hline 45 & 1 \\
\hline 67 & 1 \\
\hline 68 & 1 \\
\hline 45 & 1 \\
\hline 71 & 1 \\
\hline 75 & 1 \\
\hline 82 & 1 \\
\hline 84 & 1 \\
\hline 86 & 1 \\
\hline 86 & 1 \\
\hline 88 & 1 \\
\hline 100 & 1 \\
\hline
\end{tabular}

\subsubsection{Twitter}

When comparing the quantization tables of the original images to the non stego images downloaded from Twitter (produced in steps 1 through 3 of the method used), 37 out of 40 are the same value. The quality factor frequency for non stego images downloaded from twitter are in Table 15.

Table 15: Quality Factor for Twitter Images

\begin{tabular}{|l|l|}
\hline $\begin{array}{l}\text { Quality } \\
\text { Factor }\end{array}$ & Frequency \\
\hline 80 & 10 \\
\hline
\end{tabular}




\begin{tabular}{|l|l|}
\hline 90 & 5 \\
\hline 92 & 4 \\
\hline 75 & 3 \\
\hline 85 & 3 \\
\hline 93 & 2 \\
\hline 99 & 2 \\
\hline 45 & 1 \\
\hline 67 & 1 \\
\hline 82 & 1 \\
\hline 84 & 1 \\
\hline 85 & 1 \\
\hline 86 & 1 \\
\hline 88 & 1 \\
\hline 100 & 1 \\
\hline
\end{tabular}

\subsubsection{Reddit}

The same analysis that was conducted on non stego images from Twitter was conducted on Reddit non stego images. For Reddit, there is similar performance with 35 out of 40 being the same. The frequency of the table quality for non stego images downloaded from Reddit are shown in Table 16. 
Table 16: Quality Factor Frequency for Reddit Images

\begin{tabular}{|l|l|}
\hline $\begin{array}{l}\text { Quality } \\
\text { Factor }\end{array}$ & Frequency \\
\hline 80 & 9 \\
\hline 75 & 8 \\
\hline 90 & 4 \\
\hline 92 & 4 \\
\hline 85 & 3 \\
\hline 45 & 1 \\
\hline 67 & 1 \\
\hline 68 & 1 \\
\hline 71 & 1 \\
\hline 82 & 1 \\
\hline 84 & 1 \\
\hline 85 & 1 \\
\hline 86 & 1 \\
\hline 88 & 1 \\
\hline 99 & 1 \\
\hline 100 & 1 \\
\hline
\end{tabular}




\subsubsection{Steganography Tools}

All the images produced by the OutGuess tool had a JPEG quality factor of 75 and the corresponding quantization tables pictured in figure 22. The same tables were produced regardless if the cover image was downloaded from Twitter or Reddit (80 images total).

\begin{tabular}{|c|c|c|c|c|c|c|c|}
\hline 8 & 6 & 6 & 7 & 6 & 5 & 8 & 7 \\
\hline 7 & 7 & 9 & 9 & 8 & 10 & 12 & 20 \\
\hline 13 & 12 & 11 & 11 & 12 & 25 & 18 & 19 \\
\hline 15 & 20 & 29 & 26 & 31 & 30 & 29 & 26 \\
\hline 28 & 28 & 32 & 36 & 46 & 39 & 32 & 34 \\
\hline 44 & 35 & 28 & 28 & 40 & 55 & 41 & 44 \\
\hline 48 & 49 & 52 & 52 & 52 & 31 & 39 & 57 \\
\hline 61 & 56 & 50 & 60 & 46 & 51 & 52 & 50 \\
\hline
\end{tabular}

\begin{tabular}{|c|c|c|c|c|c|c|c|}
\hline 9 & 9 & 9 & 12 & 11 & 12 & 24 & 13 \\
\hline 13 & 24 & 50 & 33 & 28 & 33 & 50 & 50 \\
\hline 50 & 50 & 50 & 50 & 50 & 50 & 50 & 50 \\
\hline 50 & 50 & 50 & 50 & 50 & 50 & 50 & 50 \\
\hline 50 & 50 & 50 & 50 & 50 & 50 & 50 & 50 \\
\hline 50 & 50 & 50 & 50 & 50 & 50 & 50 & 50 \\
\hline 50 & 50 & 50 & 50 & 50 & 50 & 50 & 50 \\
\hline 50 & 50 & 50 & 50 & 50 & 50 & 50 & 50 \\
\hline
\end{tabular}

Figure 22: Table 0 and 1 for OutGuess Images [21]

All stego images produced by ExifTool and Steghide had the same quantization tables of the corresponding cover images used ( 80 pairs of images). This provides an advantage since it makes it harder to detect. When comparing the uploaded stego images to the corresponding downloaded stego images, the quantization tables are also all the same. Since DIIT produces stego images with a PNG format, quantization tables aren’t applicable.

\subsubsection{Key Takeaways}

This method of detection (using Quantization Tables) does not necessarily guarantee that image steganography is being used. It is still possible for a non stego image to have the same quantization matrices as a stego image. That being said, this method only useful for identifying a set of tools that were possibly used to create a stego image. It not a reliable solution to detecting image steganography itself. It's also only limited to detecting stego images that are JPEGs which is another disadvantage for the technique. 


\section{Threats to Validity}

\subsection{Construct}

The length of the embedded message used in the experiment could have affected the results. To mitigate this issue a short message was used for all images.

\subsection{Internal}

The data quality could have been affected by user errors that could have occurred during the trial (uploading the incorrect image and downloading the incorrect image are examples). This could not be totally avoided, but breaks were taken in between the testing of each combination to avoid mistakes caused by mental fatigue.

\subsection{Conclusion}

While making a conclusion, the results of related works could have affected the thought process. It could have caused an expectation of certain results while analyzing the data. Since the trials conducted for each combination were not repeated, there is a possibility of a phenomenon having different outcomes when measured more than once. This was mitigated by the pilot experiment which tested the method used for consistency.

\subsection{External}

Images used for the experiment could have not been representative of images that are regularly posted online. Since the images that were used were downloaded from google images, they could be considered a good representation.

\section{Conclusion}

In this experiment, a modified version of a method developed for posting image steganography to social media was further tested with more combinations of stego tools and social media websites. The results show that Twitter and Reddit showed some promise in serving as a medium for sharing these particular types of images. Reddit outperformed Twitter as it was always able to retain an image's integrity regardless of the size of an image or the stego tool that was used. When considering the results case study and experiment conducted in 2019 [1], and the results of 
this experiment, Steghide seems to be the best stego tool to use since it has the most recorded success.

The results of experiment have shown that some of the steganography techniques, like OutGuess, are able to be detected by the consistency in the quantization tables of the images that they produce. The other tools that were tested did not have this behavior, which makes it more difficult in using this method of detection.

When analyzing the developed method for posting stego images to social media, one of the advantages for the method is that it mitigates some of the effects of image processing on social media websites. One of the disadvantages is that parties attempting to use this method would have to post an image twice, which could lead to their act of secret communication being detected. This can be mitigated by the user by downloading images that have already been posted and using them as cover images.

\section{Future Work}

For future, more combinations of stego tools and social media websites can be tested. Reddit should especially be tested with more tools with it showing a lot of success in the experiment conducted. Other factors should also be considered for future tests such as the size of the image, the length of the message (embedding capacity), and the type of embedded data (text, video, photo). Stegware (Images embedded with Malware) and social media is another area that needs more research.

\section{References}

[1] Trotter, Lindsey Kathryn, "A case study involving creating and detecting steganographic images shared on social media sites" (2019). Graduate Theses and Dissertations. 17798 [2] J. Ning, I. Singh, H. V. Madhyastha, S. V. Krishnamurthy, G. Cao and P. Mohapatra, "Secret message sharing using online social media," 2014 IEEE Conference on Communications and Network Security, 2014, pp. 319-327

[3] "Frequently asked question about the Google+ shutdown". Google.

https://support.google.com/googlecurrents/answer/9217723?hl=en (Accessed Nov. 23, 2020) 
[4] H. Tankovska. "Most popular social networks worldwide as of January 2021, ranked by number of active users". Statista. https://www.statista.com/statistics/272014/global-socialnetworks-ranked-by-number-of-users/. (accessed Feb. 1, 2021)

[5] "Steganography". Marriam-Webster. https://www.merriam-

webster.com/dictionary/steganography. (Accessed Nov. 10, 2020)

[6] "Steghide-Documentation".Steghide. http://Steghide.sourceforge.net/documentation.php

(Accessed March 20th 2020).

[7] "Steghide Manual". Steghide.

http://steghide.sourceforge.net/documentation/manpage.php(Accessed( Mar. 20,2020)

[8] “rot13.com”. ROT13. https://rot13.com/ (Accessed Sept. 23, 2020).

[9] “Digital Invisible Ink Toolkit”. DIIT. http://diit.sourceforge.net/ (Accessed Mar. 20"th , 2020)

[10] N. Sharma and U. Batra, "A review on spatial domain technique based on image

steganography," 2017 International Conference on Computing and Communication

Technologies for Smart Nation (IC3TSN), 2017, pp. 24-27

[11] "Discrete Cosine Transform". Mathworks.

https://www.mathworks.com/help/images/discrete-cosine-transform.html (Accessed Feb. 10, 2021).

[12] "Definition of JPEG". Merriam-Webster. https://www.merriam-

webster.com/dictionary/JPEG (Accessed Jan. 15, 2021).

[13] “Cardinal Bird Wildlife Red”.Pixabay. https://pixabay.com/photos/cardinal-bird-wildlifered-5375277/ (Accessed Jan. 21, 2021).

[14] R. Brennecke, U.Burgel, G. Rippin, F. Post, H. Rupprecht, J. Meyer ,“Comparison of image compression viability for lossy and lossless JPEG and Wavelet data reduction in coronary angiography”.NIH. https://pubmed.ncbi.nlm.nih.gov/11495503/(Accessed Mar. 28, 2021) [15] "Definition of Cryptography". Merriam-Webster. https://www.merriam-

webster.com/dictionary/cryptography (Accessed Jan. 10, 2020)

[16] "Rail Fence Cipher". DCODE. https://www.dcode.fr/rail-fence-cipher (Accessed Sept. 28, 2020).

[17] Coles, Kendall, "Evaluating Social Media as a Medium of Private Communication through the Use of Steganographic Images", unpublished. 
[18] H. Tankovska, "Media Usage in an Internet Minute as of August 2020”. Statista. https://www.statista.com/statistics/195140/new-user-generated-content-uploaded-by-users-perminute/ (Accessed Feb. 12, 2021)

[19] T. Penvy, J. Fridrich, "Estimation of Primary Quantization Matrix for Steganalysis of Double-Compressed JPEG Images". Binghamton.

https://ws2.binghamton.edu/fridrich/Research/paper_3_color.pdf (Accessed Apr. 1, 2021) [20] Kornblum, Jessse, "Using JPEG quantization tables to identify imagery processed by software".

https://dl.acm.org/doi/abs/10.1016/j.diin.2008.05.004 (Accessed Mar. 20, 2021)

[21] "Forensically". Photo Forensics. https://29a.ch/photo-forensics/\#jpeg-data (Accessed Mar. 29, 2021).

[22] "Woodburn Hall West Virginia". Pixabay. https://pixabay.com/photos/woodburn-hall-westvirginia-1057364/ (Accessed Apr. 28, 2021).

[23] Sayood, Khalid, "Introduction to Data Compression".

ScienceDirect.https://www.sciencedirect.com/topics/computer-science/lossless-compression

(Accessed Mar. 11, 2021).

[24] K. Szcypiorski, "StegHash: New Method for Information Hiding in Open Social Networks". Arvix. https://arxiv.org/ftp/arxiv/papers/1611/1611.00306.pdf (Accessed Feb. 14, 2021) [25] "FreeBSD Manual Pages". Freebsd.

https://www.freebsd.org/cgi/man.cgi?query=outguess $+\&$ apropos=0\&sektion=0\&manpath=Free $\underline{\text { BSD }+ \text { Ports }+5.1-\text { RELEASE\&format }=\text { html }}($ Accessed Mar. 12, 2021)

[26] Hussain, Mehdi, “Image steganography in spatial domain: A survey”. ScienceDirect. https://www.sciencedirect.com/science/article/abs/pii/S092359651830256X (Accessed Mar. 12, 2021)

[27] "ExifTool by Phil Harvey". ExifTool. https://exiftool.org/ (Accessed Mar 1. 2021)

[28] "Fawn Deer West Virginia". Pixabay. https://pixabay.com/photos/fawn-deer-west-virginia4490208/ (Accessed Apr. 28, 2021). 